\title{
Nanomaterial-based aptamer sensors for arsenic detection
}

Kang Mao ${ }^{\mathrm{a}}$, Hua Zhang ${ }^{\mathrm{a}^{*}}$, Zhenglu Wang ${ }^{\mathrm{b}}$, Haorui Cao ${ }^{\mathrm{a}}$, Kuankuan Zhang ${ }^{\mathrm{a}}$, Xiqing $\mathrm{Li}^{\mathrm{b}}$ and Zhugen $\mathrm{Yang}^{\mathrm{c}}{ }^{*}$

${ }^{a}$ State Key Laboratory of Environmental Geochemistry, Institute of Geochemistry, Chinese Academy of Sciences, Guiyang, 550002, China

${ }^{b}$ Laboratory for Earth Surface Processes, College of Urban and Environmental Sciences, Peking University, Beijing, 100871, China

${ }^{c}$ Cranfield Water Science Institute, Cranfield University, Cranfield, MK43 OAL, United Kingdom

Corresponding Email: zhanghua@vip.gyig.ac.cn (H. Z.) and zhugen.yang@cranfield.ac.uk (Z.Y.) 


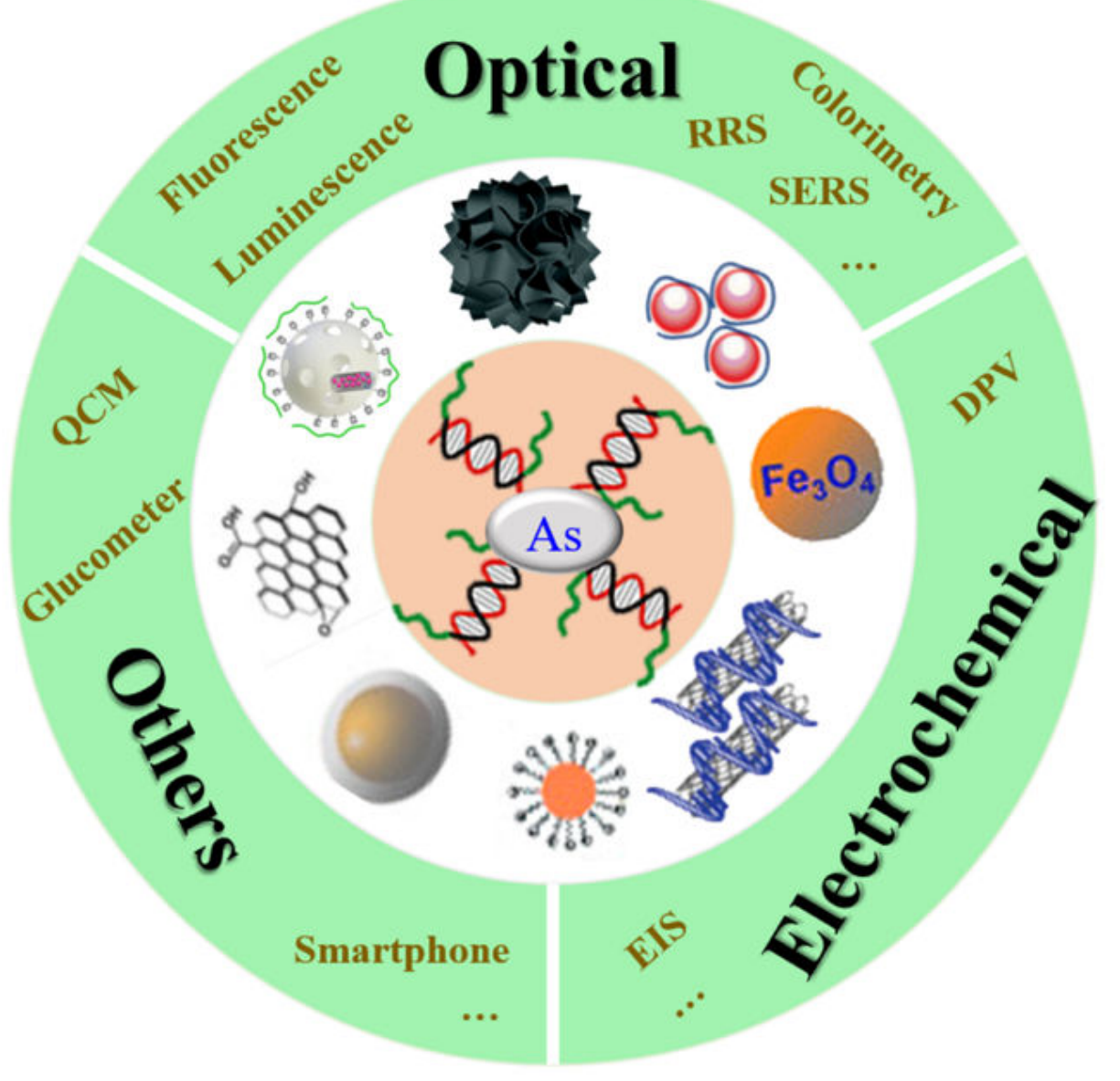

Graphical abstract 


\begin{abstract}
Arsenic (As) is a highly toxic contaminant in the environment and a serious carcinogen for the human being. The toxicity of arsenic significantly threatens environmental and human health. The effective removing technology for arsenic remains challenging, and one of the reasons is due to the lack of powerful detection method in the complex environmental matrix. There is thus an urgent need to develop novel analytical methods for arsenic, preferably with the potential for the field-testing. To combat arsenic pollution and maintain a healthy environment and eco-system, many advanced analytical methods have been developed for arsenic detection in various samples. Among these strategies, biosensors hold great promise for rapid detection of arsenic, in particular, nanomaterials-based aptamer sensors have attracted significant attention due to their simplicity, high sensitivity and rapidness. In this paper, we reviewed the recent development and promising applications of aptamer sensors (aptasensors) based-on nanomaterial for arsenic detection, in particular with emphasis on the works using optical and electrochemical technologies. We also discussed the recent novel technology in aptasensors development for arsenic detection, including nucleic acid amplification for signal enhancement and device integration for the portability of arsenic sensors. We are hoping this review could inspire further researches in developing novel nanotechnologies based aptasensors for possible on-site detection of arsenic.
\end{abstract}

Keywords: Arsenic, aptasensors, nanomaterials, optical and electrochemical sensors, signal amplification

\title{
Content
}

\section{Introduction}

2. Arsenic

\subsection{Sources of arsenic}

\subsection{Chemistry and toxicity of arsenic}

\section{Aptamer-based arsenic biosensors}

\subsection{Nanomaterial-based optical aptasensors}

\subsubsection{Fluorescent arsenic aptasensor}

3.1.2. Colorimetric arsenic aptasensors

3.1.3. SERS-based arsenic aptasensors

3.1.4. Chemiluminescence-based arsenic aptasensors

3.1.5. Other optical signal-based arsenic aptasensors

3.2. Electrochemical aptasensor for arsenic detection

4. Nucleic acid amplification for signal enhancement of arsenic detection

5. Integrated aptasensors device for arsenic detection

6. Conclusion, Challenge, and Perspectives 


\section{Introduction}

Arsenic (As) is a widely distributed and highly toxic heavy metal in the environment. The contamination of arsenic in the environment, especially in groundwater, which is one of the most significant burden for safe drinking water sources, has posed a global threat to public health. The presence of arsenic in groundwater has been reported in many areas of the world, such as Argentina, Australia, Bangladesh, Cambodia, Canada, Chile, China, Ghana, Germany, Hungary, India, Japan, Laos, Mexico, Nepal, Pakistan, Poland, Romania, Taiwan, Thailand, UK, USA, and Vietnam (Basu et al. 2014; Hsueh et al. 1998; Tseng et al. 2015)(Farzin et al. 2017; Kaur et al. 2015)(Devi et al. 2019; Ma et al. 2015; Melamed 2005; Sadee et al. 2015). Rapid and reliable methods for arsenic determination is becoming an urgent task. So far, many determination strategies have been published for the analysis of arsenic in environmental matrices, including Inductively Coupled Plasma Mass Spectrometry (ICP-MS) (Liu et al. 2013)(Jackson et al. 2015), Atomic Absorption Spectroscopy (AAS) (Linhart et al. 2016), and Atomic Fluorescence Spectrometry (AFS) (Chen et al. 2014; Fang et al. 2014). However, most of these advanced instrument-dependent arsenic determination techniques are commonly time-consuming and highly expensive with well-trained personnel (Farzin et al. 2017; Kaur et al. 2015). To address these issues, the aptamer sensor (aptasensor) has been introduced as an alternative and effective method, which proves to be an excellent candidate for simple, rapid, accurate, and sensitive analytical methods for arsenic detection (Iliuk et al. 2011; Kaur et al. 2015).

The aptamer is a single-stranded nucleic acid sequence, which has been reported since1990 (Ellington and Szostak 1990; Robertson and Joyce 1990; Tuerk and Gold 1990). The conformation of aptamer may change into secondary and tertiary structures by binding certain target, leading to the signal change, which can be transduced and read with an physiochemical format. This characteristics make it a very promising receptor for the development of biosensors. To screen aptamer with excellent affinity to a certain target, systematic evolution of ligands by exponential enrichment (SELEX) technology has been performed. The identified aptamer can play a role of bio-receptor to specifically recognize the analyte during construct an aptasensor (Jiang et al. 2012; Zhan et al. 2016). Aptamer has the capacity of binding to a range of targets, including ions (Farzin et al. 2017; Freeman et al. 2011; Zhan et al. 2016), small molecules (Li et al. 2019b), peptides (Centi et al. 2007), cells (Guo et al. 2017), tissues (Li et al. 2019a) and even organisms(Iliuk et al. 2011). These nucleic acid probes offered advantages compared with conventional ligands, such as high binding affinity similar to an antibody that could lead to the excellent specificity, selectivity and stability, and lack of immunogenicity and toxicity. Furthermore, the facile synthesis and ease of functionalization made them ideal candidates for the recognition of targets in complex samples (Akki and Werth 2018; Iliuk et al. 2011). Thus, aptasensors have been extensively explored for quantitative detection of a range of targets including arsenic.

As mentioned above, arsenic is a widely distributed and highly toxic heavy metal in the environment, especially in groundwater. Arsenic would enter into the human body system via the food chain due to that groundwater is one of the most important and stable sources of drinking water. Hence, a simple, rapid, and cheap arsenic 
determination is becoming an urgent task for analytical science research and environmental monitoring. However, most of the traditional determination methods, such as ICP-MS, AAS, and AFS, are commonly not only expensive instruments requirement, but also time-consuming and need professionally trained operators. As an alternative method, aptamer sensors as a new analytical platform for the detection of arsenic have been reported in the past decade. For a better understanding of this novel research field, we will systemically review the advances in arsenic aptasensors development since 2009 and hopefully this review could inspire further researches in developing novel nanomaterial-based aptasensors for future arsenic on-site detection.

In this paper, we started by introducing the arsenic source, chemistry, and toxicity, followed by the comprehensive discussion of the recent development in all aptasensorsbased arsenic detection since they were successfully screened in 2009. Nanomaterialbased arsenic aptasensors were classified according to their signal readout techniques, mainly including optical and electrochemical assays. The optical aptasensors included fluorescence, colorimetry, Surface-enhanced Raman Spectroscopy (SERS) and others. Furthermore, we highlighted several arsenic aptasensors, which were devoted to signal amplification for higher sensitivity and device integration for better portability and miniaturization. Finally, we summarized the review and gave our insights on the perspectives on the nanomaterial-based aptasensors.

\section{Arsenic}

Arsenic has attracted great attention worldwide due to high toxicity and abundance in the environment including the atmosphere, water, soil, and vegetation (Priyadarshni et al. 2018; Sadee et al. 2015). Although Arsenic has four oxidation states ( $-3,+3,0$, and +5$)$ in the environment, the most found oxidation states are trivalent arsenite and pentavalent arsenate in water samples (Ge et al. 2018; Hao et al. 2015). Many diseases including cancer, skin damage, circulatory system disease, nervous system disease, respiratory system disease, and cardiovascular problems are closely associated with the continuous intake of As (III) (Antonova and Zakharova 2016; Ge et al. 2018; Sadee et al. 2015). Both World Health Organization (WHO) and Environmental Protection Agency have provided a provisional guideline concentration of $10 \mu \mathrm{g} \mathrm{L} \mathrm{L}^{-1}(133 \mathrm{nM})$ for maximum arsenic concertation in groundwater (Gupta et al. 2016; Moghimi et al. 2015; Mulvihill et al. 2008).

\subsection{Sources of arsenic}

As a naturally distributed element, arsenic has been widely found in the soil, minerals, water, atmosphere, and even biosphere (Kaur et al. 2015; Smedley and Kinniburgh 2002). Although arsenic rarely has an occurrence in pure form in nature, it exists as main compounds over 200 minerals, including elemental, arsenide, sulfides, oxides, arsenates, and arsenites (Liu and Liu 2014). In fact, the commonly identified As-bearing minerals are the most important source of arsenic and the most common arsenic minerals are ore minerals or their alterative products (Basu et al. 2014; Kim et al. 2009). In addition to the minerals, it was suggested that the arsenic concentration in the earth's crust should be taken into consideration (Basu et al. 2014). Although it has been reported that arsenic concentrations are low in the atmosphere, they are usually 
increased through intake from fossil fuel combustion, industrial operations (smelting), and volcanic eruption, which could have a significant influence on atmosphere environment (Basu et al. 2014; Hao et al. 2015).

\subsection{Chemistry and toxicity of arsenic}

The arsenic element has the valence configuration of $3 d^{10} 4 s^{2} 4 p^{3}$ and herein exists four main oxidation forms, $-3,0,+3$, and +5 . Among the four major oxidation, -3 and 0 compounds prevail only in stronger reducing condition and herein arsenic is mostly found trivalent and pentavalent arsenic in an inorganic form in natural water (Smedley and Kinniburgh 2002). The toxicity of arsenic changes with the chemical states ranging from essentially non-hazardous to excessively hazardous, depending on (a) oxidation state and (b) the groups bound to the arsenic. Redox potential and $\mathrm{pH}$ have the most significant effect on controlling arsenic speciation. For example, although $\mathrm{AsH}_{3}$ (arsine, the hydride) is regarded as the most hazardous species while arsenosugars and arsenobetaine are non-hazardous (Basu et al. 2014). Trivalent inorganic arsenic is much more hazardous than pentavalent inorganic arsenic, and pentavalent organo-compounds are less hazardous than inorganic arsenic. Previous reports demonstrated that methylated trivalent arsenic species may be more hazardous than inorganic arsenic compounds (Styblo et al. 2000) and are certainly more hazardous than pentavalent organic arsenic (Basu et al. 2014).

Arsenic has a carcinogen effect on human beings' health by acting as a promoter for cancer development (Basu et al. 2014; Shen et al. 2013). Exposure to inorganic arsenic is relative to a variety of internal cancers, such as liver, lung, bladders, and skin along with diabetes (Basu et al. 2014). It has been confirmed as a teratogen due to its capability of crossing the placental membrane into the unborn babies' metabolic system. It was well-known for a cumulative substance by passing out of the body through urine, finger, skin, hair, and toe-nails (Basu et al. 2014). Arsenic may cause ischemic heart problem and cardio-vascular disease (Basu et al. 2014; Hsueh et al. 1998; Tseng et al. 2015). Some researchers also demonstrated that Alzheimer's disease was relative to anthropogenic arsenic (Cöl et al. 1999; Dani 2010).

Arsenic chemistry has been manipulated in biochemistry process. For example, trivalent arsenic being soft would prefer the -SH groups of different enzymes resulting in inhibiting the enzymatic procedure (Basu et al. 2014). Actually, as one of hazardous substance, arsenic could mimic the functions of "useful" bio-metals and it replaces the other metals in all kinds of cellular activities, thus causing serious malfunctions of different vital cellular processes (Shen et al. 2013)(Hu et al. 1995; Salazar et al. 2004; Yih and Lee 2000). For example, the chemistry character of arsenic is similar to phosphorus due to that structure of arsenate is similar to phosphate, thus it could substitute a phosphate group in adenosine triphosphate (ATP) composing a hydrolyzed arsenate ester of adenosine diphosphate (ADP). It makes uncoupling of phosphorylation and destruction of necessary metabolic activities (Basu et al. 2014).

\section{Aptamer-based arsenic biosensors}

A biosensor is a small device with a biological receptor (recognition element) that produces a certain response signal (optical, electrochemical, mass sensitive, etc.) 
collected by a physicochemical detector (transducer element) in the presence of target molecules (Alhadrami 2018; Zhang et al. 2017b). The recognition element could be biomoleclues (such as DNA, antibodies, peptide, apatamer), cells, and even microorganisms, which respond to specific targets. The transducer switches the specific biological recognition process into the desired physical and/or chemical signal. Aptasensor was named if an aptamer serves as the recognition element in biosensor development (Dolatabadi et al. 2011; Ezzati Nazhad Dolatabadi and de la Guardia 2014; Jamali et al. 2014).

For getting better analytical performance of the determination methods, chemical labeling or modification of oligonucleotides with reporter molecules could be employed without decreasing the affinity to analytes (Ebrahimi et al. 2013; Zhang et al. 2015). Furthermore, aptamers could be developed to withstand repeated cycles of denaturation and renaturation, which have great potential for reuse. Additionally, aptamers could be easily modified to develop a large number of analytical applications (Ping et al. 2012). Since (Kim et al. 2009) the arsenic aptamer was successfully screened using SELEX in 2009, many novel aptamer-based analytical methods have been reported on arsenic detection, including electrochemical, optical, and other measurements (Iliuk et al. 2011; Zhan et al. 2016)(Yuan et al. 2019).

\subsection{Nanomaterial-based optical aptasensors}

Optical aptasensors based on nanomaterials use nanomaterial/nanoparticle to physically measure the signal with the aptamer for recognition events (Moghimi et al. 2015). Optical signal detection methods, such as fluorescence, colorimetry, Surfaceenhanced Raman scattering (SERS), luminescence/chemiluminescence (CL), and Surface Plasmon Resonance (SPR) have been widely used for signal collection on aptasensors and nanosensors due to their excellent sensitivity and friendly use (Feng et al. 2014; Golub et al. 2009; Liu et al. 2010; Wang et al. 2010)(Devi et al. 2019). In this section, to better understand nanomaterial-based arsenic optical aptasensors, the highlights and prominent examples were discussed, according to a variety of optical signal readout methods, with a focus on fluorescence, colorimetry, SERS, chemiluminescence (Tab. 1).

Tab. 1. Available optical methods based arsenic aptasensors.

\begin{tabular}{|c|c|c|c|c|}
\hline Signal & Nanomaterial & $\begin{array}{l}\text { Range } \\
(\mathrm{nM})\end{array}$ & $\begin{array}{l}\text { LOD } \\
(\mathbf{n M})\end{array}$ & Ref. \\
\hline Fluorescence & $\mathrm{Fe}_{3} \mathrm{O}_{4}$ & $\begin{array}{l}50- \\
8.0 \times 10^{2}\end{array}$ & 50 & $\begin{array}{l}\text { (Liu and Liu } \\
\text { 2014) }\end{array}$ \\
\hline Fluorescence & QDs & $\begin{array}{l}10^{-2}- \\
10^{3}\end{array}$ & $\begin{array}{l}1.3 \times 10^{-} \\
-\end{array}$ & $\begin{array}{l}\text { (Ensafi et al. } \\
\text { 2016) }\end{array}$ \\
\hline Fluorescence & QDs & $\begin{array}{l}13.3- \\
2.0 \times 10^{3}\end{array}$ & 2.7 & $\begin{array}{l}\text { (Zhang et al. } \\
\text { 2017a) }\end{array}$ \\
\hline Fluorescence & MSNs & $\begin{array}{l}53.2- \\
7.98 \times 10^{2}\end{array}$ & 11.97 & $\begin{array}{l}\text { (Oroval et } \\
\text { al. 2017) }\end{array}$ \\
\hline Fluorescence & SNPs & $2-50$ & 0.45 & (Taghdisi et \\
\hline
\end{tabular}




\begin{tabular}{|c|c|c|c|c|}
\hline \multirow[b]{2}{*}{ Fluorescence } & \multirow[b]{2}{*}{ - } & \multirow[b]{2}{*}{$0.13-1.3 \times 10^{5}$} & \multicolumn{2}{|r|}{ al. 2018) } \\
\hline & & & $\begin{array}{l}6.7 \times 10^{-} \\
2\end{array}$ & $\begin{array}{l}\text { (Pan et al. } \\
2018 \text { ) }\end{array}$ \\
\hline Fluorescence & $\begin{array}{l}\text { nagnetic } \\
\text { eads }\end{array}$ & $\begin{array}{l}.0 \times 10^{-2}- \\
.0 \times 10^{3}\end{array}$ & $2.0 \times 10^{-3}$ & $\begin{array}{l}\text { (Zeng et al. } \\
2019 \text { ) }\end{array}$ \\
\hline Colorimetry & AuNPs & $\begin{array}{l}1.3 \times 10^{2}- \\
4.0 \times 10^{4}\end{array}$ & 70.49 & $\begin{array}{l}\text { (Wu et al. } \\
2012 b)\end{array}$ \\
\hline Colorimetry & AuNPs & $\begin{array}{l}13.3- \\
2.0 \times 10^{4}\end{array}$ & 7.98 & $\begin{array}{l}(\mathrm{Wu} \text { et al. } \\
2012 \mathrm{a})\end{array}$ \\
\hline Colorimetry & - & $\begin{array}{l}1.3 \times 10^{2}- \\
2.7 \times 10^{4}\end{array}$ & 79.8 & $\begin{array}{l}\text { (Wu et al. } \\
2013)\end{array}$ \\
\hline Colorimetry & AuNPs & $\begin{array}{l}16.8- \\
2.7 \times 10^{3}\end{array}$ & 16.8 & (Yu 2014) \\
\hline Colorimetry & AgNPs & $\begin{array}{l}6.7 \times 10^{2}- \\
9.3 \times 10^{3}\end{array}$ & 79.8 & $\begin{array}{l}\text { (Divsar et al. } \\
2015 \text { ) }\end{array}$ \\
\hline Colorimetry & AuNPs & $\begin{array}{l}13.3- \\
1.3 \times 10^{3}\end{array}$ & 16.9 & $\begin{array}{l}\text { (Thao } \\
\text { Nguyen et } \\
\text { al. 2018) }\end{array}$ \\
\hline SERS & Au@AgNPs & $\begin{array}{l}1.3- \\
1.3 \times 10^{3}\end{array}$ & 0.78 & $\begin{array}{l}\text { (Yang et al. } \\
2015 \text { ) }\end{array}$ \\
\hline SERS & Au@AgNPs & $\begin{array}{l}6.7- \\
1.3 \times 10^{2}\end{array}$ & 1.3 & $\begin{array}{l}\text { (Song et al. } \\
2016)\end{array}$ \\
\hline Luminescence & G-quadruplex & X $\quad 50-300$ & 7.6 & $\begin{array}{l}\text { (Lin et al. } \\
2017)\end{array}$ \\
\hline Electrochemiluminescence & $\mathrm{Au}-\mathrm{g}-\mathrm{C}_{3} \mathrm{~N}_{4}$ & $\begin{array}{l}1.3 \times 10^{-5}- \\
1.33 \times 10^{2}\end{array}$ & $\begin{array}{l}9.3 \times 10^{-} \\
6\end{array}$ & $\begin{array}{l}\text { (Liang et al. } \\
\text { 2018) }\end{array}$ \\
\hline RRS & - & $\begin{array}{l}1.3- \\
2.7 \times 10^{3}\end{array}$ & 2.7 & $\begin{array}{l}\text { (Wu et al. } \\
2012 \mathrm{c})\end{array}$ \\
\hline RRS & AuNPs & $\begin{array}{l}50.54- \\
3.1 \times 10^{3}\end{array}$ & 25.3 & $\begin{array}{l}\text { (Tang et al. } \\
\text { 2014) }\end{array}$ \\
\hline RS & AuNPs & $\begin{array}{l}13.3- \\
2.0 \times 10^{4}\end{array}$ & 10.2 & $\begin{array}{l}(\mathrm{Wu} \text { et al. } \\
2012 \mathrm{a})\end{array}$ \\
\hline
\end{tabular}

\subsubsection{Fluorescent arsenic aptasensor}

Nanoparticles that can provide fluorescence signals in the development of aptasensors have many advantages. The recognition process between aptamers and analytes occur when the conformation changes take place. Under the construction of sensors, such conformational changes could result in changing the emission properties of fluorescent systems owing to altering the original environment of fluorophores or nanomaterials. Fluorescent analytical strategies hold a huge potential in the quantitative analysis due to the maneuverable ways with wide response range and excellent sensitivity. Considering the limitations of traditional organic dyes, such as narrow absorption, broad emission and photo-bleaching, functionalized nanomaterials with fluorescence emission that could solve the problems have been widely employed to 
explore these processes using fluorescence resonance energy transfer (FRET) or electron transfer quenching as photo-physical probing mechanisms, by which new horizons have opened for signal assisted by aptasensor (Golub et al. 2009; Jamali et al. 2014; Liu et al. 2010; Wang et al. 2010). Fluorescent DNA sensors often made full use of FRET for determination of all kinds of targets like small molecules, DNA/RNA, peptides, and proteins (Iliuk et al. 2011; Saha et al. 2017; Zhang et al. 2019). If we want to develop an excellent FRET-based aptasensor, the critical consideration is the donor and acceptor fluorophore optimization. There are many acceptor and donor fluorophores available for FRET-based sensors, such as organic dyes, auto-fluorescent proteins and inorganic nanostructures (Zhang et al. 2019). Many fluorescent inorganic reporters, including gold nanoparticles (AuNPs), two-dimension materials like graphene oxide (GO), and quantum dots (QDs) have been widely used as acceptor and donor fluorophore owing to their wonderful fluorescent properties, such as high spectral resolution and resistance to photobleaching (D et al. 2017; Dolatabadi et al. 2011; Jamali et al. 2014; Vaishanav et al. 2017).

The first paper on arsenic fluorescent aptasensor was published in 2014 (Liu and Liu 2014), which was a DNA-based biosensor for arsenate detection using DNA adsorption by magnetic beads. As shown in Fig. 1A, magnetic beads adsorbed fluorescentlylabeled oligonucleotides through the phosphate backbone and resulted in fluorescence quenching. Then, arsenate has displaced the adsorbed oligonucleotides because of a higher affinity towards arsenate to increase fluorescence. The proposed sensor allows for a limit of detection (LOD) of arsenate as low as $300 \mathrm{nM}$. Notably, this assay was a novel way to use DNA for target recognition through its phosphate instead of the bases.

Additionally, organic-inorganic hybrid nanomaterials were also proposed as efficient ways of determination of a variety of targets. However, the combination of nanomaterials and aptamer to detect heavy metals are still scarce within this framework. As an inorganic scaffold, mesoporous silica nanoparticles with a diameter of $100 \mathrm{~nm}$ were widely employed as capped materials. Herein, the combination of mesoporous silica nanoparticles with aptamers (Oroval et al. 2017), has been designed as a novel fluorescence sensor for As (III) determination spanning a dynamic range from $53.2 \mathrm{nM}$ to $798 \mathrm{nM}$ with a LOD at $11.97 \mathrm{nM}$. The proposed sensing principle was showed in Fig. 1B: The pores of the inorganic support were modified by Rhodamine B and then the external surface was functionalized with aminopropyl moieties. The final capped solid was made through the introduction of the aptamer. When As (III) was introduced, it would induce unblocking of the pores by the aptamer displacement from the surface of mesoporous silica nanoparticles with subsequent dye delivery. Compared with other potential nanomaterials used in sensing protocols, mesoporous silica nanoparticles are one of the most promising supports because of their remarkable properties such as high inner surface area, flexible surface-modification chemistry and easily functionalized with (bio) chemical or supramolecular ensembles. 


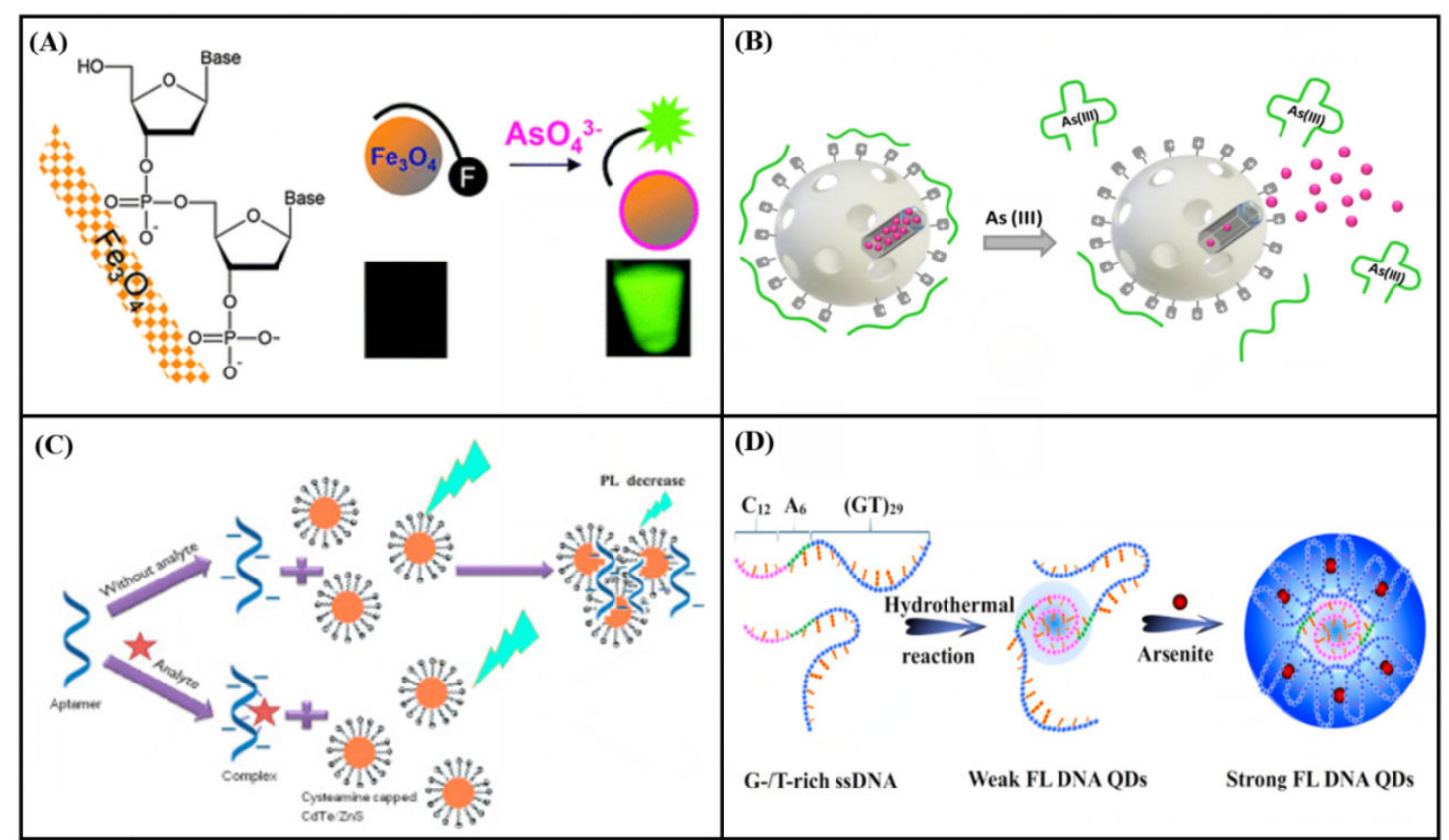

Fig. 1. Principles of representative fluorescence-based arsenic aptasensor (A) DNA adsorption by magnetic iron oxide nanoparticles and its application for arsenate detection(Liu and Liu 2014); (B) Fluorescence aptasenors of As (III) using silica nanoparticles (Oroval et al. 2017); (C)The fluorescence quenching analysis(Ensafi et al. 2016) and (D) the fluorescence enhancement analysis (Zhang et al. 2017a) based QDs aptasensor for arsenite determination.

Another arsenic aptasensor based on silica nanoparticles (SNPs) was reported by Taghdisi and co-workers (Taghdisi et al. 2018). Specifically, they fabricated and characterized a fluorescence biosensor for As (III) determination using the conformational change of target induced by the biotin and FAM-modification complementary strand of aptamer, silica nanoparticles modified by streptavidin (SNPsStreptavidin) and unlabeled aptamer. When the As (III) was introduced, the aptamer released its complementary strand of DNA, forming a hairpin structure on the surface of SNPs-Streptavidin and resulting in a remarkable fluorescent response signal. In the absence of As (III), a weak fluorescence response was collected due to that the aptamer was hybridized with its complementary strand of DNA. This biosensor can detect As (III) as low as $0.45 \mathrm{nM}$.

Although organic fluorescent probes were useful in arsenic species assay with excellent analytical performance, photobleaching and the stability issue limited their wide applications in arsenic determination. Alternatively, quantum dots (QDs) have been developed aiming to solve the problems, which are fluorescent semiconductors containing elements of Groups II-VI, III-V, and IV-VI (Freeman et al. 2013; Lesiak et al. 2019). QDs provide fluorescent signals in the construction of aptasensors and hold many excellent features over traditional dyes, including broad absorption spectra, high quantum yield and exceptional photochemical stability (Jamali et al. 2014; Jamieson et al. 2007). QDs are therefore extensively used for the construction of biosensing platforms (Frasco and Chaniotakis 2009). For instance, Ensafi et al (Ensafi 
et al. 2016) have developed a CdTe/ZnS QDs aggregation-based fluorimeter aptasensor for As (III). In their report, the aptamer was designed to aggregate cationic cysteaminestabilized CdTe/ZnS QDs, which led to fluorescence quenching. When was introduced As (III), the complex between the aptamer and As (III) prevented aggregation of the QDs (Fig. 1C). Therefore, depending upon the As (III) concentration, the QDs fluorescence was enhanced due to the de-aggregation. The fluorescence analysis held a promising LOD of $1.3 \mathrm{pM}$ with a dynamic range from $1.0 \times 10^{-2}$ to $1.0 \times 10^{3} \mathrm{nM}$. The proposed QDs based aptasensor has advantages such as high sensitivity and selectivity, compared with aptasensors for As (III) detection using conventional dyes.

Apart from fluorescence quenching strategy, the fluorescence enhancement of DNA QDs based aptasensor was also investigated and applied for arsenic determination. Zhang et al (Zhang et al. 2017a) reported an arsenite detection strategy based on the fluorescence enhancement of DNA QDs. In their work, the synthesized DNA QDs using G/T-rich ssDNA showed special optical properties, and acquired the basic structure and biological activities of ssDNA precursors, which made the QDs selectively bind with arsenite, driving the $(\mathrm{GT})_{29}$ region towards the conformation switching and form the well-ordered assembly (Fig. 1D). They speculated that the strong inter-molecule interaction and efficient stacking of base pairs stiffen the assembly structure, blocked non-radiative relaxation channels, populated radiative decay, and thus made the assembly highly emissive as a new fluorescence center. The certain fluorescence enhancement induced by arsenite promotes QDs as light-up probes for determination of arsenite. A very good linear relationship was demonstrated between fluorescence intensity and logarithmic arsenite concentration from $1 \mu \mathrm{g} \mathrm{L}^{-1}$ to $150 \mu \mathrm{g} \mathrm{L} \mathrm{L}^{-1}$ with a LOD at $0.2 \mu \mathrm{g} \mathrm{\textrm {L } ^ { - 1 }}$.

\subsubsection{Colorimetric arsenic aptasensors}

Nobel metal nanomaterials, especially gold nanoparticles (AuNPs) and silver nanoparticles (AgNPs), owing to good optical properties depending on different size and distance, are excellent materials for colorimetric analysis. Taking AuNPs as an example, they hold unique optical and electrical properties, including high absorption coefficient, highly SPR, scattering flux, luminescence and conductivity (Gong et al. 2017). For instance, the color of AuNPs was highly sensitive to its aggregation and dispersion because of inter-particle plasmon coupling changing and then led to surface plasmon band shift (Gong et al. 2017). Another excellent character of AuNPs is the large specific surface area (Priyadarshni et al. 2018). The large surface-to-volume ratio could adsorb numerous bio-macromolecules onto their surfaces. The special characters of AuNPs made it become a good signal transducer for aptasensor construction (Hutter and Maysinger 2013; Jeong et al. 2014). Moreover, the extinction coefficient of AuNPs is much higher than organic dyes (more than 1000 times), which give excellent sensitivity for colorimetric biosensor based AuNPs (Ghosh and Pal 2007; Rex et al. 2006). As a consequence, nanoparticles could be acted as a novel signal indicator of color analysis through assembly and disassembly. Herein, colorimetric aptamer biosensors are a good choice for simple detection of arsenic in some certain matrix (Gong et al. 2017; Priyadarshni et al. 2018). 
First colorimetric arsenic aptasensor has been reported by $\mathrm{Wu}$ and co-workers $(\mathrm{Wu}$ et al. 2012b). In their work, cationic polymer (poly-diallyldimethylammonium, PDDA) aggregated AuNPs and caused an obvious color variance because As (III) selectively interacted with its aptamer due to the formation of As-aptamer complex, which made colorimetric detection for arsenic with high sensitivity (Fig. 2A). In the same year, using the same aggregation principle but different polymer (cetyltrimethylammonium bromide, CTAB)-induced aggregation of AuNPs (Fig. 2B), they reported another aptamer-based biosensor for As (III) detection. The dynamic range spanned from 1 to $1500 \mathrm{ppb}$ with the limitation of detection of $0.6 \mathrm{ppb}$ for color analysis and $40 \mathrm{ppb}$ for naked-eye detection, respectively (Wu et al. 2012a). Following the same strategy, Thao et al (Thao Nguyen et al. 2018) also developed a novel biosensor based on CTAB and AuNPs for colorimetric assay of ppb levels of As (III) with a LOD of $16.9 \mathrm{ppb}$ in real samples.

In addition to the polymer induced aggregation of AuNPs, Zhou's group also used the salt-induced aggregation of AuNPs (classical aptamer-based AuNPs colorimetric method) for As (III) detection (Zhan et al. 2014). In this study, as shown in Fig. 2C, an arsenic aptamer was employed as the probe with AuNPs as a colorimetric signal. When As (III) was absent in the solution, AuNPs were wrapped by aptamer and therefore were stable even when a high concentration of $\mathrm{NaCl}$ was in the solution, showing a red color solution. On the contrary, when introduced As (III), the AuNPs were easy to aggregate due to the formation of the As-aptamer complex, showing a blue color solution. Through monitoring the color variance, the rapid colorimetric detection methods for As (III) a dynamic range from 1.26 to $200 \mu \mathrm{g} \mathrm{L}^{-1}$ and a LOD of $1.26 \mu \mathrm{g} \mathrm{L}^{-1}$ was demonstrated. However, AuNPs-based sensors are dependent on salt-induced aggregation, which seems to make them more susceptible to interference by environmental matrices. Natural matrices are typically diluted in a buffer before sensing, the matrices with high salt concentrations shall be very careful when performing this assay.

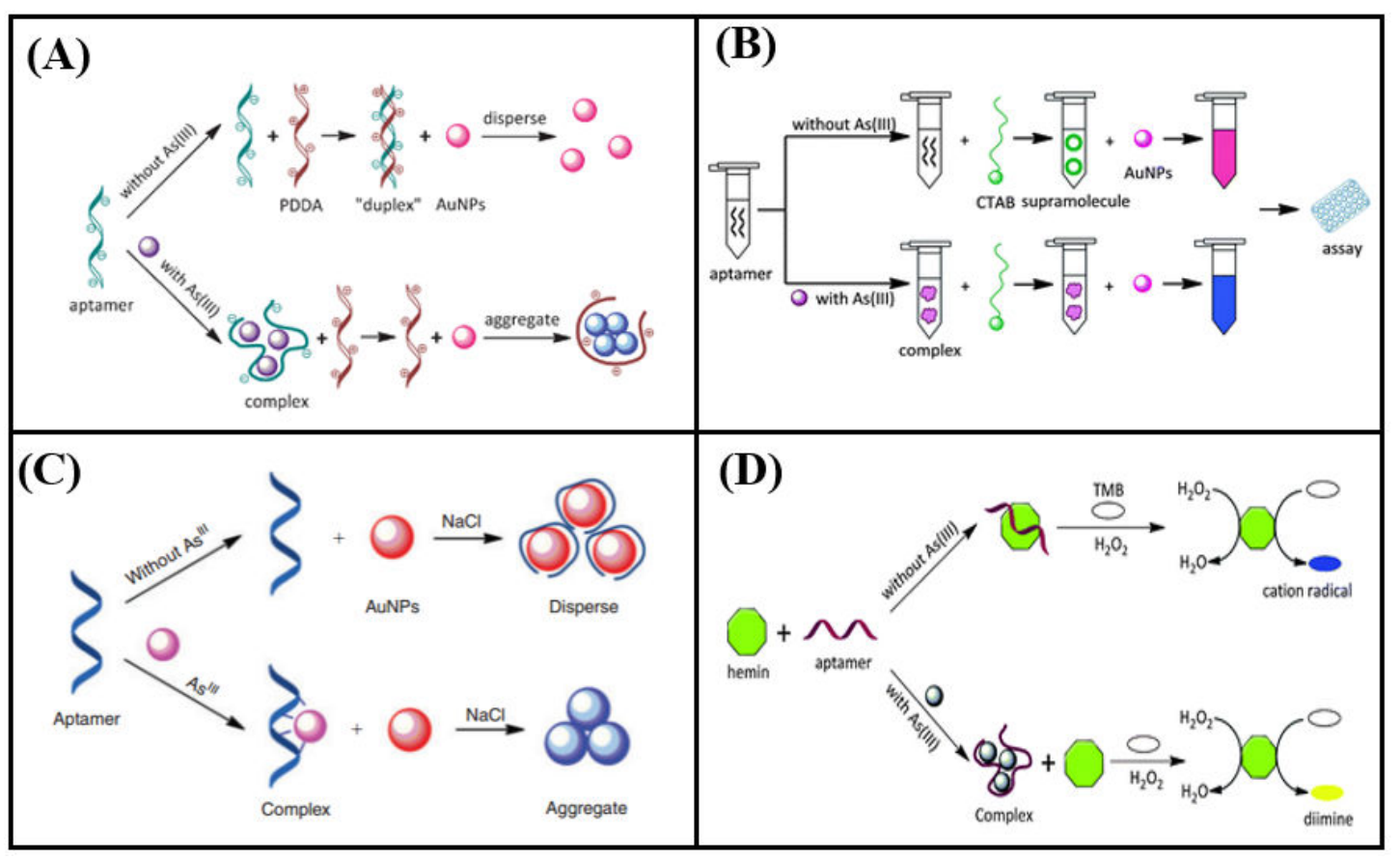


Fig. 2. Principles of representative colorimetry-based arsenic aptasensor techniques. (A) Cationic polymers and aptamers mediated aggregation of AuNPs for colorimetric detection of As (III) in aqueous solution(Wu et al. 2012b); (B) Ultrasensitive aptamer biosensor for As (III) detection in aqueous solution based on surfactant-induced aggregation of AuNPs (Wu et al. 2012a); (C) Aptasensor for As (III) detection in aqueous solution based on cationic salt-induced aggregation of AuNPs (Zhan et al. 2014); (D) Regulation of hemin peroxidase catalytic activity by As-binding aptamers for colorimetric detection of As (III)(Wu et al. 2013).

In addition to AuNPs, other important noble metal nanomaterial-silver nanoparticles (AgNPs) has also been used for arsenic detection. Divsar et al (Divsar et al. 2015) prepared AgNPs modified by aptamer (Apt-AgNPs) and used them in colorimetric determination of As (III). In their work, As (III) could selectively recognize with AptAgNPs for the formation of As (III)-Apt-AgNPs complex and cause an obvious decrease in peak intensity $\left(\lambda_{\max }=403 \mathrm{~nm}\right)$, which can be proportional to As (III) concentration. Additionally, a combination of a central composite design optimization method and response surface methodology was applied to optimize the efficiency of As (III) analysis in this experiment. The linear range of the colorimetric biosensor held a wide scope of As (III) concentration from 50 to $700 \mathrm{ppb}$ with a LOD of $6 \mathrm{ppb}$.

Additionally, DNAzymes is an example of allosteric aptamers which have been applied for biosensors development. They can serve as either recognition elements or signal readouts in these sensing platforms (Bohunicky and Mousa 2011). Among these DNAzymes, one significant type is the G-quadruplex DNAzyme with peroxidase activity that made of repetitive G-rich and hemin like quadruplex motifs. When introduced hemin, it could bind with rich G-rich DNA sequence and then form Gquadruplex-hemin complexes that demonstrate peroxidize activity as horseradish peroxidize. Herein, horseradish peroxidises mimicking DNAzymes can fabricate different colorimetric biosensors. On the basis of this mechanism, a new colorimetric aptasensor for As (III) determination was designed by the combination of aptamer and G-quadruplex DNAzyme (Wu et al. 2013). In this work, as shown in Fig. $2 \mathrm{D}$, the catalytic activity of high concentration of hemin was temporarily inhibited by As-aptamer complex, and it recovered when introduced As (III) because of the exhaustion of arsenic aptamers, thus subsequent 3,3',5,5'-tetramethylbenzidine (TMB) could be oxidized completely and led to an obvious increase of UV-vis spectra intensity, which enabled a LOD of $6 \mu \mathrm{g} \mathrm{L}{ }^{-1}$ As.

\subsubsection{SERS-based arsenic aptasensors}

Surface-enhanced Raman scattering (SERS) is a promising analytical technology with extremely high sensitivity due to huge electromagnetic enhancement induced by localized surface plasmon resonance of specific nanomaterial surfaces ( $\mathrm{Li}$ et al. 2017; Moskovits 1985; Sharma et al. 2012). SERS holds excellent characteristics, such as excellent sensitivity, high-resolution spectroscopic bands, low photo-bleaching and an extensive range of excitation wavelengths. As one of the most sensitive spectroscopic methods, SERS technology is extensively applied in environmental analysis at the 
ultra-trace inorganic and organic small molecules, DNA/RNA, peptides/proteins, cells and even organisms since its emergence in the 1980s (Chen and Choo 2008; Fleischmann et al. 1974; Jeanmaire and Van Duyne 1977). We have also developed a SERS analytical method based on 'turn-off' signal for sensitive determination of methamphetamine, which showed the superiority of aptasensor for illicit drug detection (Mao et al. 2018).

The SERS technique has a huge potential for in-situ detection of arsenic species onsite, especially using hand-held Raman spectrometers (Hao et al. 2015). The first aptasensors for As (III) detection with SERS was reported by Yang and co-workers (Yang et al. 2015). The mechanism was an aggregation of functioned $\mathrm{Au} / \mathrm{Ag}$ nanoparticles induced by the target for formation the SERS hot-spot areas that made the Raman spectra a huge enhancement. Using the similar principle, we also proposed a highly selective and sensitive analytical method for As (III) determination based on SERS technique and aptamer (Song et al. 2016). In our work, to acquire the excellent SERS substrate, Au@Ag were synthesized through seeds growth. The synthesized Au@Ag not only showed high SERS efficiency but also held well-dispersed characteristics. This biosensor held a linear rang from 0.5 to $10 \mu \mathrm{g} \mathrm{L}^{-1}$ and the LOD at $0.1 \mu \mathrm{g} \mathrm{L}{ }^{-1}$. Therefore, the target induced Au@Ag aggregation for SERS is likely to offer the tremendous possibility of As (III) determination on-site in practical applications.

However, there are only several reports about developing SERS analysis for arsenic in the past years due to many challenges remains during practical application of the SERS technique. Some important factors, such as nanostructure characters and surface chemistry of the SERS substrate, aggregation extent of Ag/Au NPs, impurities and matrix effect of sample chemistry, and analytical conditions, have a significant influence on the SERS spectra, quantification accuracy and minim detectable concentration of arsenic. More attention should be paid to the construction of stable SERS substrates which enables to quantify arsenic with excellent sensitivity, reproducibility, and stability. The previously published literatures showed that the SERS technique, especially in conjunction with commercial and portable Raman instrument, has a promising analytical strategy for on-site detection of arsenic.

\subsubsection{Chemiluminescence-based arsenic aptasensors}

As one of the attractive analytical strategies, chemiluminescence analysis has some advantages, such as simple manipulation, fast response, high sensitivity, and low expense. However, only few chemiluminescence sensors were developed for arsenic determination so far. Lin et al (Lin et al. 2017) used iridium (III) complex-based Gquadruplex for As (III) determination. The principle of the assay was in the following: The hairpin structure of DNA sequence with two loops (in a single strand status) contained As (III) aptamer sequence. At first, the G-rich sequence was partially locked to the aptamer region through base pairs. The G-rich DNA was chosen because a long G-rich sequence could give rise to a loose G-quadruplex morphology, causing falsepositive results due to the premature formation of the G-quadruplex structure even without the target. When introduced As (III), the complementary DNA sequence was released because of the formation of As-aptamer complex. The released G-quadruplex 
sequence that folded into a G-quadruplex structure, which was recognized through the G-quadruplex-selective iridium (III) complex with a luminescence enhancement signal. The assay achieved a minimum detectable concentrations of $7.6 \mathrm{nM}$.

Another example is that Liang and co-workers (Liang et al. 2018) made full use of the electrochemiluminescence of $\mathrm{Au}-\mathrm{g}-\mathrm{C}_{3} \mathrm{~N}_{4}$ nanosheets and developed an ultrasensitive arsenite determination based on the cooperative quenching effect of arsenite and $\mathrm{Ru}(\mathrm{bpy})_{3}{ }^{2+}$. In this study, according to the principle of cooperative quenching of electrochemiluminescence emission of Au-g- $\mathrm{C}_{3} \mathrm{~N}_{4}$ using As (III) and $\mathrm{Ru}(\mathrm{bpy})_{3}{ }^{2+}$, a new ratiometric electrochemiluminescence indicator was constructed for ultratrace As (III) determination, meanwhile producing a novel electrochemiluminescence signal of $\mathrm{Ru}(\mathrm{bpy})_{3}{ }^{2+}$ with increased intensity. Based on dual quenching effect of $\mathrm{As}$ (III) and $\mathrm{Ru}(\mathrm{bpy})_{3}{ }^{2+}$ coupled with production of the second electrochemiluminescence signal of $\mathrm{Ru}(\mathrm{bpy})_{3}{ }^{2+}$, the sensitivity and selectivity for detecting As (III) were tremendous enhancement with a LOD of $7.0 \times 10^{-4} \mathrm{ppt}$, ranging from $1.0 \times 10^{-3} \mathrm{ppt}$ to $10 \mathrm{ppb}$.

\subsubsection{Other optical signal-based arsenic aptasensors}

Some special optical signal has been used to develop arsenic aptasensor, such as Resonance scattering (RS) and Resonance Rayleigh scattering (RRS) (Tang et al. 2014; Wu et al. 2012a; Wu et al. 2012c). For example, Zhou's group developed an RRS assay for As (III) depending on assembled nanoparticles by aptamers and crystal violet (Wu et al. 2012c). Before As(III) determination, different nanoparticles with different sizes were firstly assembled through controlling concentration of As-binding aptamers in crystal violet solutions. Experimental characterizations of scanning probe microscopy and photon correlation spectroscopy confirmed that the size of nanoparticles indeed changed when the addition of As (III), which led to a huge change in the RRS intensity. When introduced $100 \mu \mathrm{g} \mathrm{L}^{-1}$ As (III), the maximum decrease ratio of the RRS intensity was acquired for large nanoparticles assembled from $200 \mathrm{nM}$ of aptamers and crystal violet, where the nanoparticles' average diameter decreased from $273 \mathrm{~nm}$ to $168 \mathrm{~nm}$. Considering small nanoparticles, a maximum increase in the ratio of RRS intensity was got when aptamer concentration was more than $600 \mathrm{nM}$. Combination of an RRS analysis and the above nanoparticles as the recognition element, an efficient aptasensor has been designed for the determination of As (III). The present analytical platform held a dynamic range from 0.1 to $200 \mu \mathrm{g} \mathrm{L}^{-1}$ with LOD at $0.2 \mu \mathrm{g} \mathrm{L}^{-1}$.

In summary, fluorescence, colorimetry, SERS, and chemiluminescence are four kinds of widely used analytical methods for arsenic aptasensors. Among these optical methods, fluorescence analysis, including both unlabeled and labeled modes, has become one of the most extensively applied signal transduction modes; color analysis is the simplest biosensing process and could be distinguished by the naked eye. Herein, some color aptamer biosensors for arsenic are widely constructed, mainly involving noble metal nanoparticles and formation of Horseradish peroxidase-DNAzyme with hemin. SERS and chemiluminescence have also been applied in the last several years considering its extensive calibration ranges and simple instrument system. 


\subsection{Electrochemical aptasensor for arsenic detection}

Electrochemical aptasensor is a small equipment that assembles one or more biological material/nanomaterial to an electrode transducer and holds some advantages over the optical sensor (Saei et al. 2013). Compeered to optical biosensor, electrochemical aptasensors have a huge possibility for target analysis on-site due to the easy integration with electronic device (Kempahanumakkagari et al. 2017). They required less quantity of target molecules for determination because of the feasibility of combing them to the target-binding aptamer and the biosensing amplifying procedure (Kempahanumakkagari et al. 2017). Moreover, the determination of targets without any fluorescent labels cut down the expense of the instrument and made them more reusable by cleaning the certain targets (Saei et al. 2013; Zuo et al. 2007). Recently, a few electrochemical aptasensors were reported by utilizing electrochemical impedance spectroscopy (EIS), differential pulse voltammetry (DPV), and other electrochemical signals as novel arsenic analytical strategies (listed in Tab. 2). For example, Baghbaderani and Noorbakhsh (Baghbaderani and Noorbakhsh 2019) constructed several electrochemical signal-based aptasensors for As (III) assay. For example, they made use of chitosan-Nafion (Chit-Naf) compound as an excellent conductive surface platform and a signal amplification process based novel carbon nanotube to design an unlabeled impedimetric aptasensor for As (III) determination with a high sensitivity (Fig. 3A). The EIS experimental results demonstrated that glassy carbon electrode (GCE) modified by Chit-Naf held higher electron transfer kinetics compared with bare GCE, GCE/Naf, and GCE/Chit electrodes, which provided huge feasibility as an effective platform for biosensor designation (Fig. 3B and Fig. 3C). In this work, according to carbon nanotube-bovine serum albumin (CNT-BSA) hybrid system, they also used a signal amplification process, which achieved a LOD at 74 pM (Fig. 3D). This protocol bears the advantages of reproducibility, selectivity, being mediator free and renewability of the biosensing interface, and is very useful for the rapdi As (III) detection in environmental samples. 
(A)
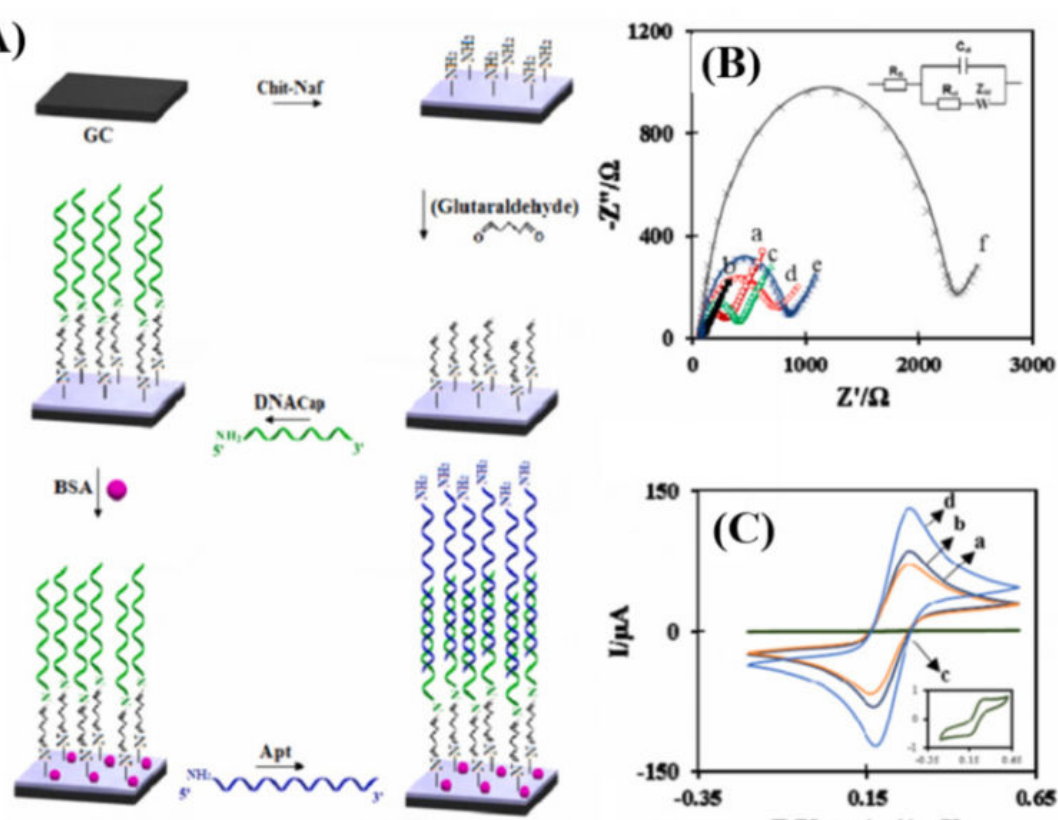

DNACap : 5:-CA-NH2-MGATGGTIT-3.

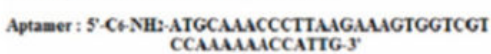

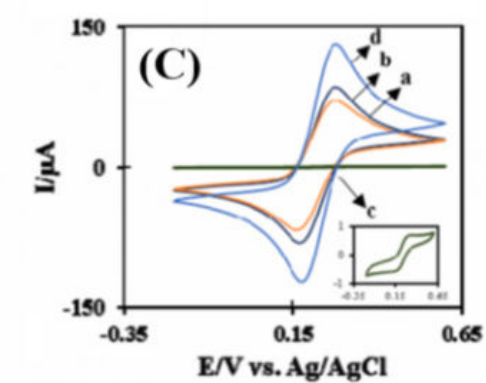

(D)

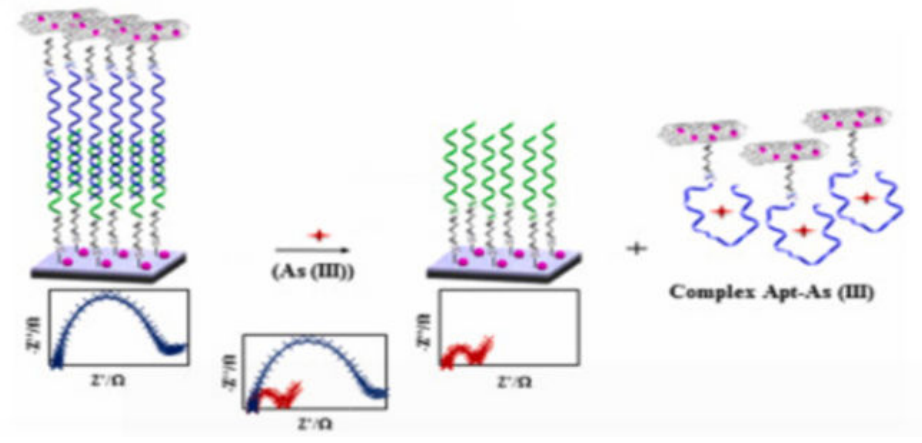

Fig. 3 Novel chitosan-Nafion composite for fabrication of highly sensitive impedimetric and colorimetric As (III) aptasensor (Baghbaderani and Noorbakhsh 2019).

Tab. 2. Available electrochemical aptasensors for detection of arsenic.

\begin{tabular}{|c|c|c|c|c|}
\hline signal & strategy & Range (nM) & LOD (nM) & Ref. \\
\hline EIS & $\overline{\mathrm{AuE}}$ & $\begin{array}{l}6.7 \times 10^{2}- \\
1.3 \times 10^{5}\end{array}$ & 800 & $\begin{array}{l}\text { (Vega-Figueroa et al } \\
2018 \text { ) }\end{array}$ \\
\hline EIS & $\mathrm{AuE}$ & $1.3-2.7 \times 10^{3}$ & 0.27 & (Gu et al. 2018) \\
\hline EIS & $\begin{array}{l}\text { 3D- } \\
\mathrm{rGO} / \mathrm{AuNPs} / \mathrm{GCE}\end{array}$ & $\begin{array}{l}5.1 \times 10^{-6}-4.0 \times 10 \\
3\end{array}$ & $\begin{array}{ll}0^{-} & 1.9 \times 10 \\
-6 & \end{array}$ & (Ensafi et al. 2018) \\
\hline EIS & Chit-Naf/GCE & $1.0-500$ & 0.78 & $\begin{array}{l}\text { (Baghbaderani and } \\
\text { Noorbakhsh 2019) }\end{array}$ \\
\hline EIS & Chit-Naf/GCE & $0.15-100$ & $7.4 \times 10^{-2}$ & $\begin{array}{l}\text { (Baghbaderani and } \\
\text { Noorbakhsh 2019) }\end{array}$ \\
\hline DPV & SWCNT/AuE & $6.7-133$ & 6.65 & (Wang et al. 2015) \\
\hline DPV & PDDA/SPCE & $0.2-100$ & 0.15 & (Cui et al. 2016) \\
\hline DPV & $\mathrm{MCH} / \mathrm{AuE}$ & $1.3-2660$ & 1.0 & (Wen et al. 2017) \\
\hline DPV & $\mathrm{GO} / \mathrm{AuE}$ & $2.7-6.7 \times 10^{3}$ & 0.77 & (Wen et al. 2018) \\
\hline
\end{tabular}




$\begin{array}{lllll}\text { Voltammetry } & \text { CFMNSs/AuE } & 1.0 \times 10^{-3}-10 & 1.0 \times 10^{-3} & \begin{array}{l}\text { (An and Jang } \\ \text { 2017) }\end{array}\end{array}$

Although most EIS-based aptasensors concentrated on protein determination (Mokhtarzadeh et al. 2015), several recent studies used EIS aptasensors for arsenic determination (Baghbaderani and Noorbakhsh 2019; Ensafi et al. 2018; Gu et al. 2018; Vega-Figueroa et al. 2018). Among these analytical methods, the recognition of arsenic through aptamer-modified gold electrodes (AuEs) changed the electron transfer resistance $\left(R_{\mathrm{ct}}\right)$ which is a corresponding correlation with the amount of arsenic. When introduced arsenic, arsenic aptamer cleaved into two or three fragments that could be self-assembled into As-binding aptamer complex (Gu et al. 2018; Vega-Figueroa et al. 2018). For instance, Vega-Figueroa et al (Vega-Figueroa et al. 2018) constructed a sensitive and selective As (III) aptasensor without any label based on the formation of supramolecular aptamer fragments/As (III) complex for arsenic determination. In this electrochemical platform, they made significant efforts to use EIS along with the aptamer to construct and explore the interfacial properties of an As (III) biosensor. The aptamer was assembled on a gold substrate, and upon binding of As (III) ( Fig. 4A), a detectable impedimetric signal change was measured due to the conformational changes of the interfacial layer. This target-induced signal based on interfacial changes was used for the selective detection of As (III), which were linearly correlated with As (III) concentration ranging from 0.05 to $10 \mathrm{ppm}$. The successful proposed technique demonstrated the feasibility of the combination of the sensitive electrochemical technique and high selective aptamer toward the target.

Glassy carbon electrode (GCE), was also commonly applied for arsenic detection with EIS (Baghbaderani and Noorbakhsh 2019; Ensafi et al. 2018). For instance, Ensafi et al (Ensafi et al. 2018) designed a novel aptasensor depending upon 3D-reduced graphene oxide modified AuNPs (3D-rGO/AuNPs) for arsenite determination (Fig. 4C). The 3D-rGO/AuNPs has been adequately characterized using different techniques and the thiolate- modified aptamer was self-assembled on the surface of GCE that it was modified with 3D-rGO/AuNPs by Au-S bond. When introduced As (III), the target and the ssDNA could form a G-quadruplex complex, which generated a hindrance for electron transfer. As a consequence, the different EIS signals of 3D-rGO/AuNPsmodified GCE was detected. Under the optimized experimental condition, the biosensor has a lower LOD of $1.4 \times 10^{-7} \mu \mathrm{g} \mathrm{\textrm {L } ^ { - 1 }}$ toward As (III) and ranges from $3.8 \times 10^{-7}$ to $3.0 \times 10^{-4} \mu \mathrm{g} \mathrm{L}^{-1}$. This biosensor demonstrated an excellent reproducibility and superior selectivity with a satisfying recovery for As (III) assay in real samples.

Another widely used electrochemical strategy for arsenic detection is Differential pulse voltammetry (DPV) (Cui et al. 2016; Wang et al. 2015; Wen et al. 2018; Wen et al. 2017). The gold electrode is usually employed for arsenic detection based on DPV. For example, Wen et al (Wen et al. 2017) designed a sensitive electrochemical arsenite aptasensor by constructing As-induced conformation change of ssDNA and electrochemical indicator-methylene blue (Fig. 4E). The aptamer firstly hybridized with a capture probe DNA on the gold electrode. Then, methylene blue was intercalated into 
the aptamer/ capture probe DNA hybrid on the electrode. When introduced As (III), it selectively bound to the aptamer, which resulted in a conformational change and the dissociation of the aptamer from the electrode into solution. As a consequence, the total amount of methylene blue on the modified electrode decreased, and this decreased the peak current of methylene blue. The assay for As (III) held a linear response from 0.1 to $200 \mathrm{ppb}$ and a LOD as low as $75 \mathrm{ppt}$.

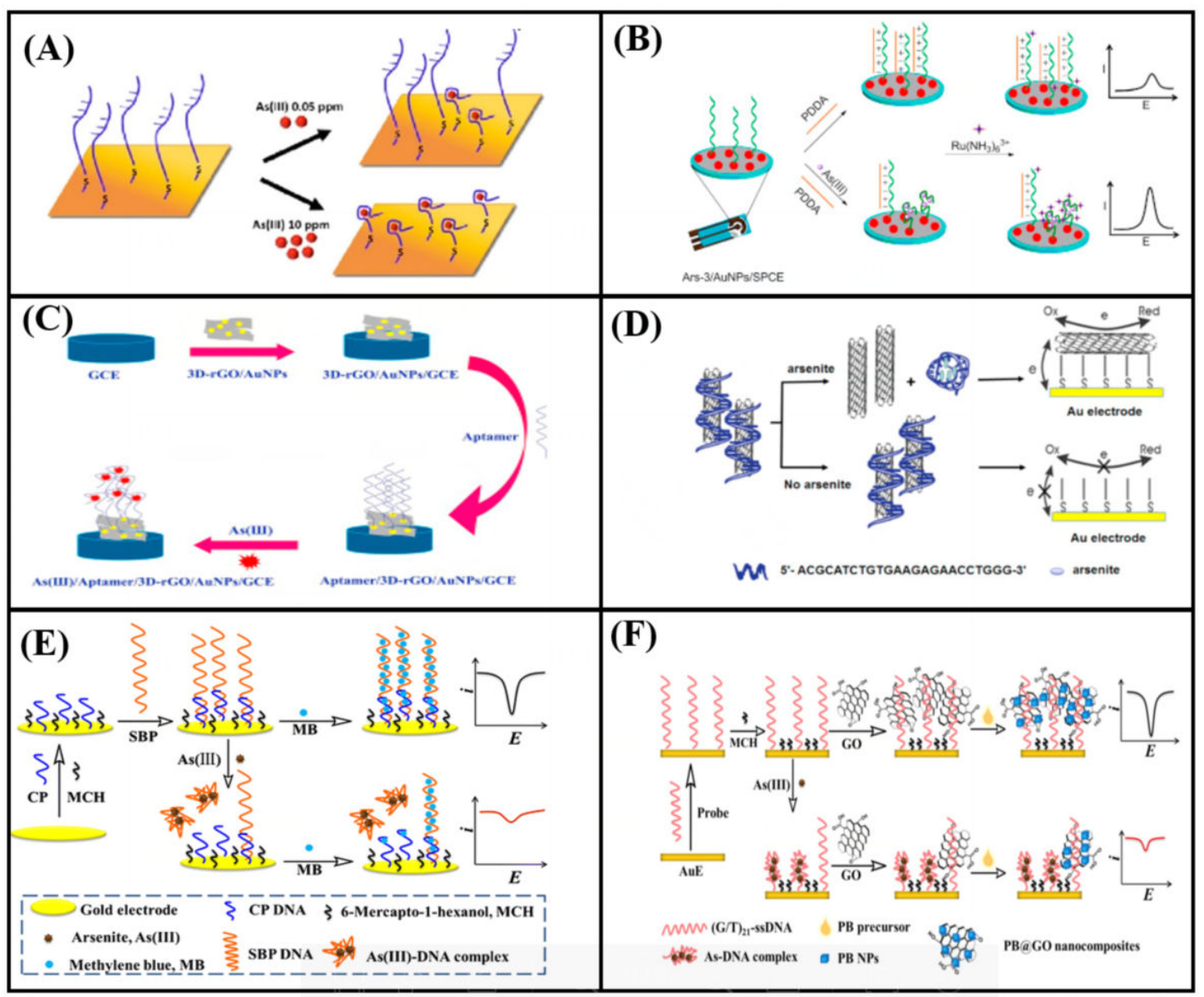

Fig. 4. Principles of representative electrochemical signal-based arsenic aptasensor techniques. (A) Aptamer-based impedimetric assay of arsenite in water (Vega-Figueroa et al. 2018); (B) Label-free signal-on aptasensor for sensitive electrochemical detection of arsenite (Cui et al. 2016); (C) A novel aptasensor based on 3D-reduced GO modified AuNPs for determination of arsenite (Ensafi et al. 2018); (D) Single strand DNA functionalized single wall carbon nanotubes as sensitive electrochemical labels for arsenite detection (Wang et al. 2015); (E) Highly sensitive voltammetric determination of arsenite by exploiting arsenite-induced conformational change of ssDNA and the electrochemical indicator methylene blue (Wen et al. 2017); (F) Electrochemical sensor for arsenite detection using GO assisted generation of prussian blue nanoparticles as enhanced signal label(Wen et al. 2018).

Cui et al (Cui et al. 2016) exploited an label-free electrochemical aptasensorfor sensitive arsenite determination with an arsenite aptamer self-assembled on a screen- 
printed carbon electrode (SPCE) by Au-S covalent binding. In this sensing platform (Fig. 4B), the aptamer could adsorb cationic poly-diallyldimethylammonium (PDDA) through electrostatic interaction to repel other cationic species. When introduced arsenite, the aptamer conformation change takes place due to the formation of aptamer/arsenite complex, which resulted in less adsorption of PDDA, and the complex could adsorb more $\left[\mathrm{Ru}\left(\mathrm{NH}_{3}\right)_{6}\right]^{3+}$ due to positive charge as an electrochemical indicator on the surface of aptasensor that generated a sensitive "turn-on" signal. The conformational changes induced by the target could be used for sensitive and selective arsenite assay ranging from $0.2 \mathrm{nM}-100 \mathrm{nM}$ and a minimum detectable concentration of $0.15 \mathrm{nM}$. The method showed wonderful specificity against other heavy metal ions due to the specific aptamer. The aptasensor based on SPCE showed the merits of simple fabrication and low cost, which had a huge potential on the arsenite assay in practical samples.

Recently, carbon nanomaterial such as GO nanosheets and CNT, have demonstrated wonderful properties for biosensor construction due to its fascinated characteristics such as stronger mechanical strength, easy modification, large surface, and excellent water dispersibility (Wang et al. 2011). Herein, some experts combined the gold electrodes and carbon nanomaterial for arsenic detection based on DPV signal (Wang et al. 2015; Wen et al. 2018). For instance, Wang et al (Wang et al. 2015) constructed an excellent electrochemical analytical method for arsenite based on the feasibility of arsenite-binding ssDNA and signal transduction of single-wall carbon nanotubes (SWCNTs) (Fig. 4D): The ssDNA/SWCNTs complexes were firstly assembled via wrapping ssDNA on the surface of SWCNTs by $\pi$-stacking. When added arsenite, arsenite could strongly bind to G/T bases of ssDNA and decrease $\pi-\pi$ interaction between SWCNTs and ssDNA, leading to certain ssDNA dissociating from the complexes. The separated SWCNTs were specifically modified on the self-assembled monolayer of the gold electrode. Then the SWCNTs onto the self-assembled monolayer-modified $\mathrm{Au}$ electrode substantially restored heterogeneous electron transfer that was almost fully blocked by the self-assembled monolayer. The assembled SWCNTs generated a sensitive and specific response transduction signal, which had a relationship with arsenite concentration. Through monitoring the SWCNTs-mediated currents, a linear response of arsenite concentration ranging from 0.5 to $10 \mu \mathrm{g} \mathrm{L}^{-1}$ with a LOD at $0.5 \mu \mathrm{g} \mathrm{L}^{-1}$ was easily achieved. This SWCNTs-based aptasensor created a simple, sensitive, nonradioactive analytical method for arsenite determination.

In order to improved the sensitivity, Wen et al (Wen et al. 2018) developed a novel electrochemical biosensor for determination of arsenite utilizing GO assisted production of Prussian blue nanoparticles (PBNPs) as enhanced redox signal (Fig. 4F). In this platform, the $(\mathrm{GT})_{21}$-ssDNA modified by thiolate was self-assembled on the surface of the gold electrode by Au-S bond. Then GO interacted with ssDNA by $\pi-\pi$ stacking interaction and facilitated the production of PBNPs on its surface as an electrochemically active indicator. If without arsenite, many GO/PBNPs were adsorbed on the surface of the electrode to generate a stronger redox signal response from PBNPs. While in existence of arsenite, (GT) ${ }_{21}$-ssDNA recognized and combined with arsenite using hydrogen bonds to form $(\mathrm{GT})_{21}$-ssDNA/arsenite complex with a frizzy structure. 
The structure switching of (GT) ${ }_{21}$-ssDNA resulted in less adsorption of GO/PBNPs on the electrode surface, leading to a reduced redox signal response. The arsenite-induced (GT) ${ }_{21}$-ssDNA conformational change was employed to determinate the concentration of arsenite with a linear range from 0.2 to $500 \mu \mathrm{g} \mathrm{L}^{-1}$ and LOD at $58 \mathrm{ng} \mathrm{L}^{-1} \mathrm{~L}$. Benefited from $(\mathrm{GT})_{21}$-ssDNA containing arsenite recognition sequence, the designed biosensor demonstrated excellent selectivity against other interfere and had a huge potential on trace arsenite detection in the environment.

An and Jang (An and Jang 2017) developed a sensitive field-effect transistor (FET)type aptasensor using carboxylic polypyrrole (CPPy)-coated flower-like $\mathrm{MoS}_{2}$ nanospheres (CFMNSs) for As (III) determination on-site (Fig. 5). As shown in Fig. 5A, they Firstly successfully fabricated CFMNSs by the vapor deposition polymerization method. Then, As (III)-binding aptamer-conjugated CFMNSs were fabricated into a liquid-ion gated FET system, resulting in an ultra-rapid response $(<1$ s) (Fig. 5B). Field-induced current changes occurred by the interaction between As (III) and aptamer, leading to obvious discrimination of As (III) at lower concentrations (1 pM). What's more, this CFMNSs-based aptasensors pecificity recognized As (III) against the other interference and precisely monitored As (III) in a mixture. This FET aptasensor could also determinate targets in river water samples. This aptasensorbased on $\mathrm{MoS}_{2}$ was a potential tool for As (III) assay and could be applied in a wide practical application. 
(A)
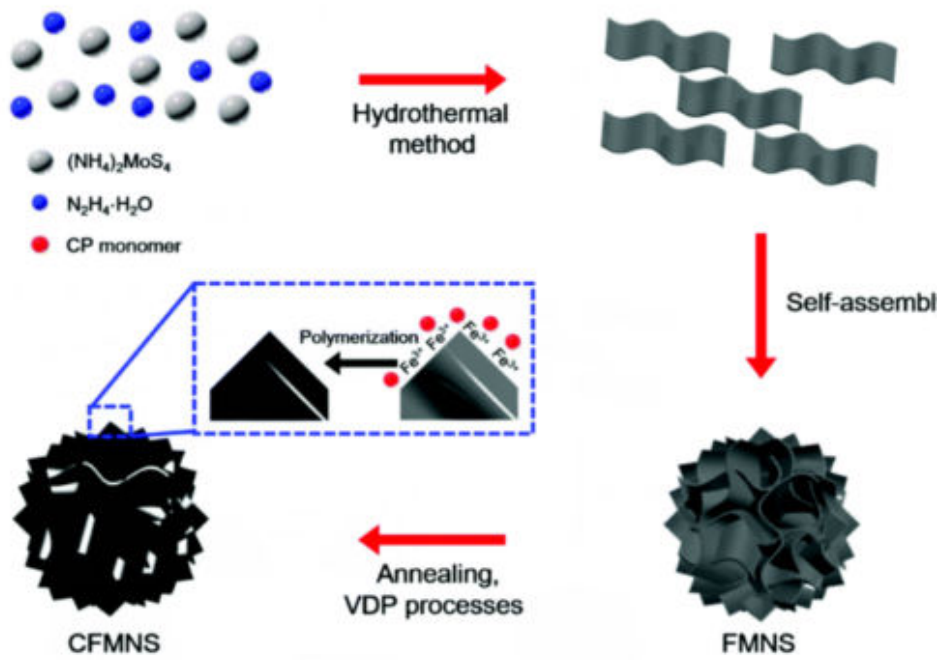

(B)
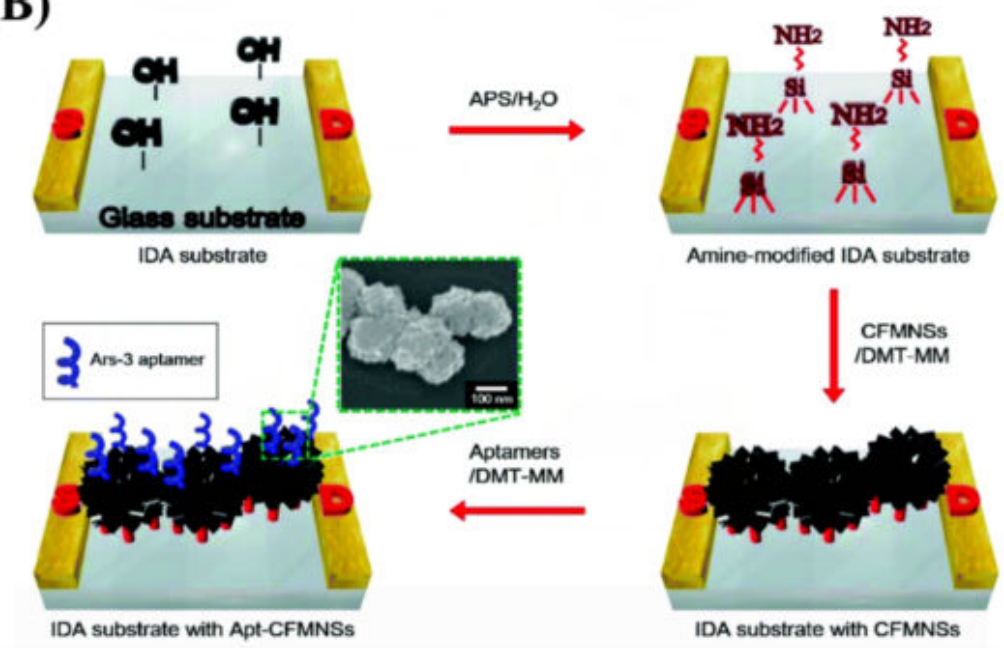

Fig. 5. Highly sensitive field-effect transistor-type aptasensor using flower-like $\mathrm{MoS}_{2}$ nanospheres for real-time detection of As (III) (An and Jang 2017).

Eectrochemical aptasensors for arsenic detection is an attractive area which has been paid more and more attention. Using advanced micro/nanomaterials, several electrochemical biosensors for arsenic were fabricated. These methods provide some new opportunities on the advantages of rapid response and low expense with high sensitivity and selectivity. In addition to much more efforts that have been devoted to constructing novel aptasensor platform, some experts tried their best to integrate the nanomaterial assembled electrode biosensor systems into portable devices.

In addition to optical and electrochemical assay, The mass sensitive method (e.g. quartz crystal microbalance (QCM)) for aptamer-based As analysis, has also been reported as label-free sensing platform by Yuan et al. (Yuan et al. 2019) in 2019. In this work, the monolayer of mercaptoethylamine was assembled to immobilize arsenite on the surface of QCM. AuNPs interacted with aptamer for amplification of the biosensor signal frequency. Arsenite firstly bound to the mercaptoethylamine on the gold surface of the QCM. When introduced AuNPs with aptamer (DNA-AuNPs), the mercaptoethylamine-As (III)-aptamer sandwich structure was produced. This increased 
the resonance frequency of the biosensor and allowed low concentration arsenite to be detected ranging from 8 to $1000 \mathrm{nM}$ with a limit of detection as low as $4.4 \mathrm{nM}$. The proposed analytical method can be used as a generic platform to detect other targets of interests such as small inorganic/organic molecules.

\section{Nucleic acid amplification for signal enhancement of arsenic detection}

In order to obtain high sensitivity for arsenic detection, researchers have developed a range of signal amplification strategy to improve the sensitivity (Pan et al. 2018). Isothermal nucleic acids amplification is a straightforward strategy of rapid and efficient nucleic acid sequences amplification at a certain temperature. A variety of amplification techniques were designed as alternatives to a polymerase chain reaction (PCR) since the early 1990s. These techniques now have widely been applied to analyze the targets such as cells, DNA/RNA, proteins/peptides, and small molecules. Amplicons generated by amplification strategy have also been employed to develop versatile nucleic acid nanomaterials for prospective applications in biosensing and bioanalysis. Aptamer integration-based single-molecule analysis has also been implemented. In this part, we will briefly review nucleic acids-based amplification technique in arsenic aptasensor.

Pan et al (Pan et al. 2018) designed an ultrasensitive fluorescence aptasensor for As (III) analysis based on an unlabeled triple-helix molecular switch and exonuclease III (Exo III)-assisted cascade target recycling amplification technique (Fig. 6A). In this work, the triple-helix molecule switching was the biosensing component and 2-amino5, 6, 7-trimethyl-1, 8-naphthyridine (ATN) was the signal indicator. The sensor exhibited a minimum detectable concentration of $5 \mathrm{ppt}$ with excellent sensitivity and selectivity. Not only that, the assembled biosensor demonstrated the feasibility of application in As (III) determination in the actual sample. These results had a huge potential in the construction of novel fluorescent biosensors for As (III) quantification in environmental water samples. Furthermore, the designed strategy could be further applied to analyze other ions with the novel molecule switching, as well as antibiotics, pesticides, and biomarkers by utilizing respective aptamers.

Using the same exonuclease III (Exo III), Zeng et al (Zeng et al. 2019) designed a fluorescence aptasensor for a low concentration of As (III) determination based on target-triggered successive signal amplification technique utilizing DNAzyme as the biocatalytic amplifier. The process was demonstrated in Fig. 6B, the specific recognition between As (III) and the aptamer could release the blocking DNA and then trigger signal amplification processes. Exo III-mediated DNA recycling digest recycling digestion process was introduced into the analytical system to produce a lot of $\mathrm{Mg}^{2+}$-dependent DNAzymes. Then the active DNAzyme with multiple turnovers could catalyze the continuous cleavage of the substrate strands functionalized by fluorophore quencher after magnetic separation, thus yielding a significant fluorescence amplification/enhancement signal for As (III) determination. Because of synergetic signal amplification of Exo III and DNAzyme, this aptasensor showed high selective detection of As (III) (LOD of $2 \mathrm{pM}$ ). The proposed biosensor also demonstrated a high selectivity toward As (III) and had been successfully employed to detect As (III) with 
satisfactory accuracy in water samples. This sensing strategy could be further fabricated as a general analytical platform for simple and rapid determination of aptamer-binding targets.

Gu et al (Gu et al. 2018) developed an electrochemical signal amplification strategy for As (III) mediated by hybridization chain reaction (HCR) and $\operatorname{Rec}_{\mathrm{f}}$ exonuclease catalytic reaction (Fig. 6C). In this sensing platform, DNA was firstly modified on the surface of gold electrodes before analysis, which generated tremendous charge-transfer resistance $\left(R_{\mathrm{ct}}\right)$. When introduction of As (III), aptamer sequence specifically bound As (III) and DNA dissociation occurred. The release of HCR product significantly decreased $R_{\mathrm{ct}}$, which was further enhanced by $\operatorname{Rec} \mathrm{J}_{\mathrm{f}}$ exonuclease catalyzed digestion. An extensive detection range from 0.1 to $200 \mathrm{ppb}$ with a LOD of $0.02 \mathrm{ppb}$ was achieved, which could be predicted that the combination of portable electrochemical instrumentation, the analytical technique was appropriate for the on-site arsenic assay.

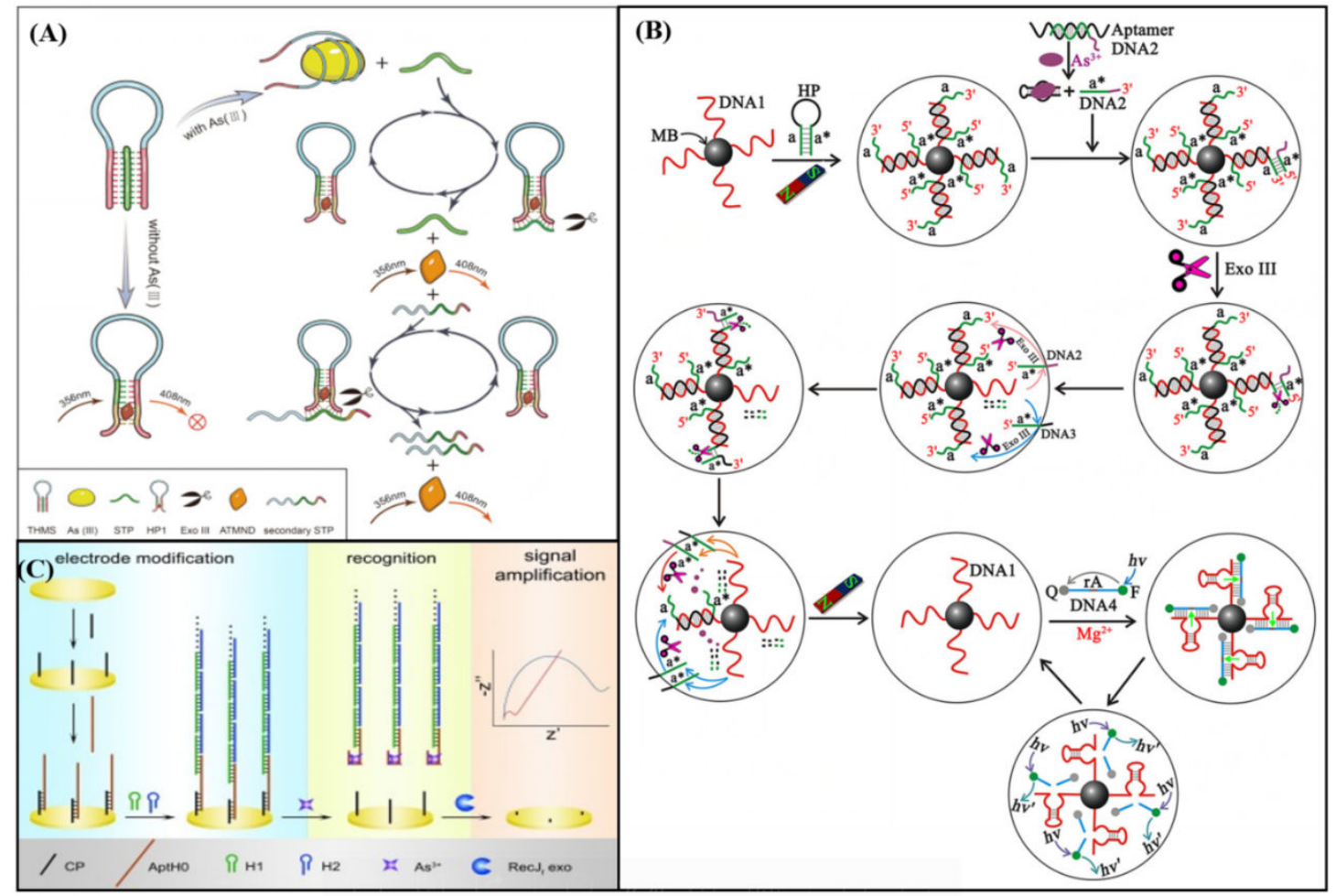

Fig. 6. Arsenic detection based on signal amplification. (A) Ultrasensitive aptamer biosensor for As (III) detection based on label-free triple-helix molecular switch and fluorescence sensing platform(Pan et al. 2018); (B) Highly sensitive aptasensor for trace As (III) detection using DNAzyme as the biocatalytic amplifier (Zeng et al. 2019); (C) Electrochemical detection of As contamination based on hybridization chain reaction and RecJf exonuclease-mediated amplification(Gu et al. 2018).

Like other metal ions and small molecules, nucleic acid amplification techniques can't be immediately applied to detect arsenic. Most of these targets use aptamer to trigger amplification for determination (Zhao et al. 2015). Herein, if we want to detect ultra-trace arsenic contamination from environmental samples, such as in drinking water, an ultrasensitive arsenic analytical method based on aptasensor using nucleic 
acid amplification would be an efficient strategy.

\section{Engineering aptasensors device for arsenic detection}

Aptasensors are usually adequately integrated with miniaturized and portable devices, such as lab on chips (Lin et al. 2017), capillary platforms, and test strips (Qi et al. 2018; Zhao et al. 2015). Commercial system and diagnostic kits give the possibility for proof of concept analysis (Chen et al. 2017; Siddiqui et al. 2018; Yang et al. 2017; Zhou and Tang 2018). The integration of aptasensor into microsystems or portable tools confers high sensitivity and promotes biosensor-based on-site assays (Yang et al. 2017). We hence discussed two successes in components integration with aptasensor for arsenic detection in 2018 (Siddiqui et al. 2018; Zhou and Tang 2018). In addition to one microfluidic chip (Lin et al. 2017), these are the only two reports on the device integration for arsenic aptasensor so far.

Zhou and Tang (Zhou and Tang 2018) have managed to make graphene oxide -gated mesoporous silica nanocontainers utilizing aptamers for arsenite assay with personal glucometer readout. In this work, the aptamer was initially bound to the mesoporous silica nanocontainers by the epoxy-amino reaction. Then, the indicator (glucose) was gated into the pores by using graphene oxide nanomaterial-based $\pi$-stacking interactions between graphene and nucleobases. When added into arsenite, graphene oxide was dissociated from MSN due to interaction between target and aptamer, thus leading to that the pore was open and then the loaded glucose was released, which could be quantified by utilizing a portable personal glucometer. According to different affinities between graphene and target for certain aptamer on the mesoporous silica nanocontainers, the amount of released glucose from the pores increased with the increasing concentrations of arsentite. Under the optimized condition, the graphene oxide-based sensing system demonstrated good personal glucometer readout signal relative to arsenite concentration within a linear range from 0.01 to $100 \mathrm{ppb}$ and a LOD of $2.3 \mathrm{ppt}$. The coefficients of variation for reproducibility of intra-assay and inter-assay were below $9.1 \%$ and $11.6 \%$, respectively. Additionally, the arsenite analysis method demonstrated a high selectivity against other ions and was employed to analyze arsenite in practical application in agreement with the gold-standard ICP-MS.

Another example is on the miniaturization of sample preparation and rapid analysis of arsenite in soil utilizing a cellphone contributed by (Siddiqui et al. 2018) (Fig. 7). With the development of computing and multitasking performances, sensors based on cellphone/smartphone have a huge potential in transferring the analytical processes in a lab to on-site analytical methods. Park et al showed the miniaturized device integration of simultaneous sample preparation and optical aptasensor for arsenic analysis based on a smartphone (Fig. 7A). The principle of As (III) detection was aggregated AuNPs induced by $\mathrm{NaCl}$ (Fig. 7B) as mentioned above. Colorimetric analysis protocol using aptamers, $\mathrm{AuNPs}$ and $\mathrm{NaCl}$ were optimized and tested on the polydimethylsiloxane (PDMS)-chip to acquire the LOD of $0.71 \mathrm{mg} \mathrm{L}^{-1}$ in the sample (Fig. 7C). The analytical performance of the system was shown by the comparative analysis of arsenic in contaminated soil with a good correlation coefficient of 0.992 with standard laboratory method. Using the Android system application on the device 
to run the experiment, the whole process from sample preparation to determination was finished within 3 hours without professional technicians. The approximate expense of device setup was evaluated about 1 USD, weight $55 \mathrm{~g}$. Herein, this method offered a portable and low-cost analytical method for arsenic analysis in the field. The combination with geometric information inside smartphones, the device would allow the determination of contaminated soils in a wide range.

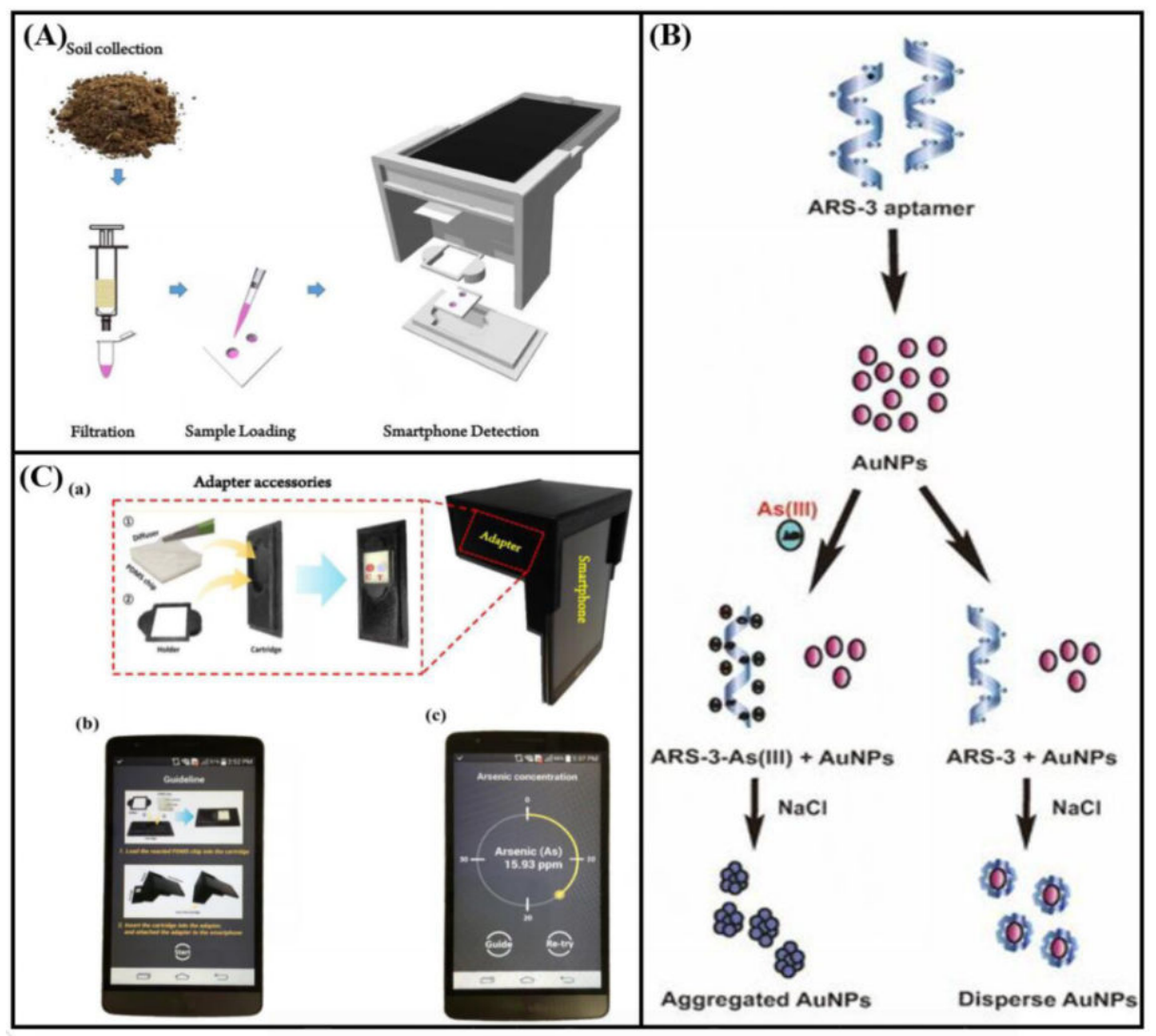

Fig. 7. (A) Schematic illustration of soil processing and smartphone-based As (III) detection procedure. (B) The principle of As (III) detection with AuNPs. (C) (a) Optical device with its accessories; (b) Screenshots of operating on an Android smartphone: Guideline of preparation for measurement of As (III) concentration; (c) The result of estimated As (III) concentration processed by Android system using colorimetric analysis.

The integration of nanomaterials-based aptasensors with commercial portable devices showed tremendous potential for construction of miniaturization biosensors for arsenic determination with fast-responses on site. Such combination had an excellent selectivity while promoting the designation and fabrication of integrated analytical methods. The applications of nanomaterials in the construction of such biosensors lead to an obvious increase in reproducibility, sensitivity, and sensibility. The ultrasensitive biosensing platform is a desirable feature of arsenic determination tools for environmental samples. Commercial portable devices using nanomaterials and nanotechnologies offered many opportunities for the construction of efficient analytical platforms. Meanwhile, the miniaturization and portability of the device are good for on- 
site detection of arsenic in a simple and low-cost way. Additionally, owing to the synergies observed when coupling certain nanomaterials, construction of appropriately integrated analytical methods may offer online or implanted arsenic determination methods that are helpful to environmental research and other fields, such as food safety analysis.

\section{Conclusion and Perspectives}

Arsenic pollution significantly threats environment and human health, and the reliable and effective monitoring of arsenic in the environment which may provide proper guidance for the development of removing strategy, however, remains a major challenge,. Aptasensors have recently been emerged as a promising tool for the rapid detection of arsenic in the environment due to the stability of aptamer. What's more, aptamer is a short DNA/RNA sequence and enables an approach from the nucleic acidbased signal amplification strategy to improve the sensitivity, for example, isothermal amplification. Aptasensors could be detected with optical, electrochemical and other technology. Fluorescence, colorimetry, SERS, and chemiluminescence are commonly used optical analytical methods for arsenic. Among these methods, fluorescent analysis has become one of the most extensive signal transduction modes due to the maneuverable ways with wide response range and excellent sensitivity. Colorimetry analysis is the simplest biosensing process and could be even read by the naked eye. We reviewed some colorimetry aptasensors for arsenic are widely constructed, mainly involving noble metal nanoparticles and the formation of Horseradish peroxidaseDNAzyme with hemin. SERS and chemiluminescence have also been employed for aptasensors due to its extensive calibration ranges and simple instrument system. Electrochemical aptasensors have a huge potential for the on-site analysis. They required less quantity of target molecules for determination because of the feasibility of combing them to target-binding aptamer and the biosensing amplifying procedure. moreover, determination of targets without any fluorescent labels cut down the expense of the instrument and made them more reusable by cleaning the certain targets.

Additionally, nanomaterials owing to their huge advantages (e.g. high surface area, tunable surface structures, and excellent optical, electrical, mechanical features) have tremendous potential in arsenic detection. The combination of nanomaterial and aptamer demonstrated a clear feasibility of a portable assay, which will facilitate sensitive and selective on-site determination of targets in the environmental samples, such as in lakes and rivers, preventing arsenic pollution.

Although currently most of the literature focused on the capability of aptasensors to sense arsenic in a wide range of matrices with minimal sample treatment, few of them have been employed in practical samples measurements due to the interference of complicated environmental matrix. In fact, the sophisticated environmental matrix in real samples has an important effect on the selectivity and sensitivity of aptasensors. The selectivity of the sensors in natural matrices has not been widely evaluated but is often relatively poor given the concentrations of analogs in natural waters. Apart from the interference from environmental samples, the aptasensor is not sensitive enough to detect the arsenic at concentrations present in natural waters. These limit the wide use 
of arsenic aptasensors for the detection of real samples. Moreover, in addition to the sensitivity and selectivity, one also need to take into account the portability, ease-ofuse, stability, robustness, reproducibility, and the cost before they can be used for the practical use or the potential for commercialization.

To overcome these obstacles, more efforts need to be dedicated to effective and selective screening aptamers for arsenic that is extremely selective and sensitive in both the bound and free states, and that undergo sufficient conformation change upon target binding either to alter agglomeration or to unbind from a complementary DNA sequence. Taking into account the rapid development of versatile nanomaterials, scientists are dedicated to exploring these nanomaterials' merits to improve analytical performance. Once we understand unknown intrinsic properties and functional moieties of nanomaterials causing the difficulty in bio-conjugation chemistry, it will be greatly benefit the construction of aptasensors. Besides, once we also successfully promote selectivity, sensitivity, robustness, stability, reusability, and reproducibility of the aptasensor, we believe that nanomaterials -based aptasensors will push forward great advances on analytical platform applied in the areas of biomedical and environmental analysis, food safety and industry.

\section{Acknowledgement}

ZY thanks UK NERC Fellowship grant (NE/R013349/2), grant from SRPe (PECRE1819/05), Royal Academy of Engineering (FoDSF $\backslash 1819 \backslash 1 \backslash 8)$ and State Key Laboratory of Environmental Geochemistry (SKLEG2019 717), CAS. We thank National Key R\&D Program of China (No. 2017YFD0800302), Natural Science Foundation of China (4157312), the National Science Foundation of China-Project of Karst Science Research Center (U1612442) and Opening Fund of the State Key Laboratory of Environmental Geochemistry (SKLEG2019606, 2019717).

\section{References}

Akki, S.U., Werth, C.J., 2018. Environ. Sci. Technol. 52(16), 8989-9007.

Alhadrami, H.A., 2018. Biotechnol. Appl. Biochem. 65(3), 497-508.

An, J.H., Jang, J., 2017. Nanoscale 9(22), 7483-7492.

Antonova, S., Zakharova, E., 2016. Electrochem. Commun. 70, 33-38.

Baghbaderani, S.S., Noorbakhsh, A., 2019. Biosens. Bioelectron. 131, 1-8.

Basu, A., Saha, D., Saha, R., Ghosh, T., Saha, B., 2014. Res. Chem. Intermediat. 40(2), 447-485.

Bohunicky, B., Mousa, S.A., 2011. Nanotechnol. Sci. Appl. 4(1), 1-10.

Cöl, M., Cöl, C., Soran, A., Sayli, B.S., Oztürk, S., 1999. Environ. Health. Persp. 107(8), 687-689.

Centi, S., Tombelli, S., Minunni, M., Mascini, M., 2007. Anal. Chem. 79(4), 1466-1473. Chen, B., Corns, W.T., Stockwell, P.B., Huang, J. H., 2014. Anal. Methods 6(18), 75547558.

Chen, L., Choo, J., 2008. Electrophoresis 29(9), 1815-1828.

Chen, Y., Fu, G., Zilberman, Y., Ruan, W., Ameri, S.K., Zhang, Y.S., Miller, E., Sonkusale, S.R., 2017. Food Control 82, 227-232. 
Cui, L., Wu, J., Ju, H., 2016. Biosens. Bioelectron. 79, 861-865.

D, P., Saini, S., Thakur, A., Kumar, B., Tyagi, S., Nayak, M.K., 2017. J. Hazard. Mater. 328, 117-126.

Dani, S.U., 2010. Sci. Total. Environ. 408(8), 1842-1846.

Devi, P., Thakur, A., Lai, R.Y., Saini, S., Jain, R., Kumar, P., 2019. TrAC Trend. Anal. Chem. 110, 97-115.

Divsar, F., Habibzadeh, K., Shariati, S., Shahriarinour, M., 2015. Anal. Methods. 7(11), 4568-4576.

Dolatabadi, J.E.N., Mashinchian, O., Ayoubi, B., Jamali, A.A., Mobed, A., Losic, D., Omidi, Y., de la Guardia, M., 2011. TrAC Trend. Anal. Chem. 30(3), 459-472.

Ebrahimi, M., Hamzeiy, H., Barar, J., Barzegari, A., Omidi, Y., 2013. Sensor Lett. 11(3), 566-570(565).

Ellington, A.D., Szostak, J.W., 1990. Nature 346(6287), 818-822.

Ensafi, A.A., Akbarian, F., Heydari-Soureshjani, E., Rezaei, B., 2018. Biosens. Bioelectron. 122, 25-31.

Ensafi, A.A., Kazemifard, N., Rezaei, B., 2016. Biosens. Bioelectron. 77, 499-504.

Ezzati Nazhad Dolatabadi, J., de la Guardia, M., 2014. Anal. Methods 6(12), 38913900.

Fang, Y., Sun, X., Yang, W., Ma, N., Xin, Z., Fu, J., Liu, X., Liu, M., Mariga, A.M., Zhu, X., Hu, Q., 2014. Food Chem. 147, 147-151.

Farzin, L., Shamsipur, M., Sheibani, S., 2017. Talanta 174, 619-627.

Feng, C., Dai, S., Wang, L., 2014. Biosens. Bioelectron. 59, 64-74.

Fleischmann, M., Hendra, P.J., McQuillan, A.J., 1974. Chem. Phys. Lett. 26(2), 163166.

Frasco, M.F., Chaniotakis, N., 2009. Sensors 9(9), 7266-7286.

Freeman, R., Girsh, J., Willner, I., 2013. ACS Appl. Mater. Inter. 5(8), 2815-2834.

Freeman, R., Liu, X., Willner, I., 2011. J. Am. Chem. Soc. 133(30), 11597-11604.

Ge, K., Liu, J., Fang, G., Wang, P., Zhang, D., Wang, S., 2018. Sensors 18(7), 2372.

Ghosh, S.K., Pal, T., 2007. Chem. Rev. 107(11), 4797-4862.

Golub, E., Pelossof, G., Freeman, R., Zhang, H., Willner, I., 2009. Anal. Chem. 81(22), 9291-9298.

Gong, L., Du, B., Pan, L., Liu, Q., Yang, K., Wang, W., Zhao, H., Wu, L., He, Y., 2017. Microchim. Acta 184(4), 1185-1190.

Gu, H., Yang, Y., Chen, F., Liu, T., Jin, J., Pan, Y., Miao, P., 2018. Chem. Eng. J. 353, 305-310.

Guo, X.L., Yuan, D.-D., Song, T., Li, X. M., 2017. Anal. Bioanal. Chem. 409(15), 37893797.

Gupta, A., Verma, N.C., Khan, S., Nandi, C.K., 2016. Biosens. Bioelectron. 81, 465472.

Hamula, C.L.A., Guthrie, J.W., Zhang, H., Li, X.F., Le, X.C., 2006. TrAC Trend. Anal. Chem. 25(7), 681-691.

Hao, J., Han, M. J., Han, S., Meng, X., Su, T.L., Wang, Q.K., 2015. J Environ Sci 36, 152-162.

Hsueh, Y.M., Wu, W.L., Huang, Y.L., Chiou, H.Y., Tseng, C.H., Chen, C.J., 1998. 
Atherosclerosis 141(2), 249-257.

Hu, J.J., Dubin, N., Kurland, D., Ma, B.L., Roush, G.C., 1995. Mutat. Res. 336(2), 193. Hutter, E., Maysinger, D., 2013. Trends Pharmacol. Sci. 34(9), 497-507.

Iliuk, A.B., Hu, L., Tao, W.A., 2011. Anal. Chem. 83(12), 4440-4452.

Jackson, B., Liba, A., Nelson, J., 2015. J. Anal. Atom Spectrom. 30(5), 1179-1183.

Jamali, A.A., Pourhassan-Moghaddam, M., Dolatabadi, J.E.N., Omidi, Y., 2014. TrAC Trend. Anal. Chem. 55, 24-42.

Jamieson, T., Bakhshi, R., Petrova, D., Pocock, R., Imani, M., Seifalian, A.M., 2007. Biomaterials 28(31), 4717-4732.

Jeanmaire, D.L., Van Duyne, R.P., 1977. J. Electroanal. Chem. 84(1), 1-20.

Jeong, E.H., Jung, G., Hong, C.A., Lee, H., 2014. Arch. Pharm. Res. 37(1), 53-59.

Jiang, B.Y., Feng, F.M., Wang, M., Li, C., Xie, J.Q., 2012. Adv. Mater. Res. 560-561, 305-308.

Kaur, H., Kumar, R., Babu, J.N., Mittal, S., 2015. Biosens. Bioelectron. 63, 533-545.

Kempahanumakkagari, S., Deep, A., Kim, K.H., Kumar Kailasa, S., Yoon, H.O., 2017. Biosens. Bioelectron. 95, 106-116.

Kim, M., Um, H.J., Bang, S., Lee, S.H., Oh, S.J., Han, J.H., Kim, K.W., Min, J., Kim, Y.H., 2009. Environ. Sci. Technol. 43(24), 9335-9340.

Lesiak, A., Drzozga, K., Cabaj, J., Bański, M., Malecha, K., Podhorodecki, A., 2019. Nanomaterials 9(2), 192.

Li, J.F., Zhang, Y.J., Ding, S.Y., Panneerselvam, R., Tian, Z.Q., 2017. Chem Rev 117(7), 5002-5069.

Li, W., Wang, S., Zhou, L., Cheng, Y., Fang, J., 2019a. Talanta 199, 634-642.

Li, Y., Sun, L., Zhao, Q., 2019b. Anal. Chem. 91(4), 2615-2619.

Liang, R.P., Yu, L.D., Tong, Y.J., Wen, S.H., Cao, S.P., Qiu, J.D., 2018. Chem. Commun. 54(99), 14001-14004.

Lin, S., Wang, W., Hu, C., Yang, G., Ko, C.-N., Ren, K., Leung, C.H., Ma, D.-L., 2017. J. Mater. Chem. B 5(3), 479-484.

Linhart, O., Smolejová, J., Červený, V., Hraníček, J., Nováková, E., Resslerová, T., Rychlovský, P., 2016. Monatshefte für Chemie - Chem 147(8), 1447-1454.

Liu, B., Liu, J., 2014. Chem. Commun. 50(62), 8568-8570.

Liu, X., Zhang, W., Hu, Y., Cheng, H., 2013. Microchem. J. 108, 38-45.

Liu, Y., Tuleouva, N., Ramanculov, E., Revzin, A., 2010. Anal. Chem. 82(19), 81318136.

Ma, J., Zhang, T., Dong, M., 2015. IEEE J. Biomedic. Health. Infor. 19(3), 986-994.

Mao, K., Zhou, Z., Han, S., Zhou, X., Hu, J., Li, X., Yang, Z., 2018. Talanta 190, $263-$ 268.

Melamed, D., 2005. Anal. Chim. Acta 532(1), 1-13.

Moghimi, N., Mohapatra, M., Leung, K.T., 2015. Anal. Chem. 87(11), 5546-5552.

Mokhtarzadeh, A., Ezzati Nazhad Dolatabadi, J., Abnous, K., de la Guardia, M., Ramezani, M., 2015. Biosens. Bioelectron. 68, 95-106.

Moskovits, M., 1985. Rev. Modern. Phys. 57(3), 783-826.

Mulvihill, M., Tao, A., Benjauthrit, K., Arnold, J., Yang, P., 2008. Angew. Chem. Int. Edit. 47(34), 6456-6460. 
Oroval, M., Coll, C., Bernardos, A., Marcos, M.D., Martínez-Máñez, R., Shchukin, D.G., Sancenón, F., 2017. ACS Appl. Mater. Inter. 9(13), 11332-11336.

Pan, J., Li, Q., Zhou, D., Chen, J., 2018. Talanta 189, 370-376.

Ping, H., Wenli, L., Jinming, L., 2012. Sensors 12(2), 1181-1193.

Priyadarshni, N., Nath, P., Nagahanumaiah, Chanda, N., 2018. ACS Sustain. Chem. Eng. 6(5), 6264-6272.

Qi, J., Li, B., Wang, X., Fu, L., Luo, L., Chen, L., 2018. Anal. Chem. 90(20), 1182711834.

Rex, M., Hernandez, F.E., Campiglia, A.D., 2006. Anal. Chem. 78(2), 445-451.

Robertson, D.L., Joyce, G.F., 1990. Nature 344(6265), 467-468.

Sadee, B., Foulkes, M.E., Hill, S.J., 2015. J Anal. Atom. Spectrom. 30(1), 102-118.

Saei, A.A., Dolatabadi, J.E.N., Najafi-Marandi, P., Abhari, A., de la Guardia, M., 2013. TrAC Trend. Anal. Chem. 42, 216-227.

Saha, J., Roy, A.D., Dey, D., Nath, J., Bhattacharjee, D., Hussain, S.A., 2017. Sensor Actuat B Chem. 241, 1014-1023.

Salazar, A.M., Calderón-Aranda, E., Cebrián, M.E., Sordo, M., Bendesky, A., GómezMuñoz, A., Acosta-Saavedra, L., Ostrosky-Wegman, P., 2004. 255(1-2), 25-31.

Sharma, B., Frontiera, R.R., Henry, A.I., Ringe, E., Van Duyne, R.P., 2012. Mater. Today 15(1-2), 16-25.

Shen, S., Li, X.-F., Cullen, W.R., Weinfeld, M., Le, X.C., 2013. Chem. Rev. 113(10), 7769-7792.

Siddiqui, M.F., Kim, S., Jeon, H., Kim, T., Joo, C., Park, S., 2018. Sensors 18(3), 777. Smedley, P.L., Kinniburgh, D.G., 2002. Appl. Geochem. 17(5), 517-568.

Song, L., Mao, K., Zhou, X., Hu, J., 2016. Talanta 146, 285-290.

Song, S., Wang, L., Li, J., Fan, C., Zhao, J., 2008. TrAC Trend. Anal. Chem. 27(2), 108-117.

Styblo, M., Razo, L.M.D., Vega, L., Germolec, D.R., Lecluyse, E.L., Hamilton, G.A., Reed, W., Wang, C., Cullen, W.R., Thomas, D.J., 2000. Arch. Toxicol. 74(6), 289-299. Taghdisi, S.M., Danesh, N.M., Ramezani, M., Sarreshtehdar Emrani, A., Abnous, K., 2018. Sensor Actuat B Chem. 256, 472-478.

Tang, M., Wen, G., Liang, A., Jiang, Z., 2014. Luminescence 29(6), 603-608.

Thao Nguyen, N.L., Park, C.Y., Park, J.P., Kailasa, S.K., Park, T.J., 2018. New J. Chem. 42(14), 11530-11538.

Tseng, C.H., Chong, C.K., Tseng, C.P., Hsueh, Y.M., Chiou, H.Y., Tseng, C.C., Chen, C.J., 2015. Toxicol. Lett. 137(1), 15-21.

Tuerk, C., Gold, L., 1990. Science 249(4968), 505-510.

Vaishanav, S.K., Korram, J., Pradhan, P., Chandraker, K., Nagwanshi, R., Ghosh, K.K., Satnami, M.L., 2017. J. Fluoresc. 27(3), 781-789.

Vega-Figueroa, K., Santillán, J., Ortiz-Gómez, V., Ortiz-Quiles, E.O., Quiñones-Colón, B.A., Castilla-Casadiego, D.A., Almodóvar, J., Bayro, M.J., Rodríguez-Martínez, J.A., Nicolau, E., 2018. ACS Omega 3(2), 1437-1444.

Wang, G., Wang, Y., Chen, L., Choo, J., 2010. Biosens. Bioelectron. 25(8), 1859-1868. Wang, Y., Li, Z., Wang, J., Li, J., Lin, Y., 2011. Trend. Biotechnol. 29(5), 205-212. Wang, Y., Wang, P., Wang, Y., He, X., Wang, K., 2015. Talanta 141, 122-127. 
Wen, S.H., Wang, Y., Yuan, Y.H., Liang, R.P., Qiu, J.D., 2018. Anal. Chim. Acta 1002, 82-89.

Wen, S., Zhang, C., Liang, R., Chi, B., Yuan, Y., Qiu, J., 2017. Microchim. Acta 184(10), 4047-4054.

Wu, Y., Liu, L., Zhan, S., Wang, F., Zhou, P., 2012a. Analyst 137(18), 4171-4178.

Wu, Y., Wang, F., Zhan, S., Liu, L., Luo, Y., Zhou, P., 2013. RSC Adv. 3(48), 2561425619.

Wu, Y., Zhan, S., Wang, F., He, L., Zhi, W., Zhou, P., 2012b. Chem. Commun. 48(37), 4459-4461.

Wu, Y., Zhan, S., Xing, H., He, L., Xu, L., Zhou, P., 2012c. Nanoscale 4(21), 68416849.

Yang, B., Chen, X., Liu, R., Liu, B., Jiang, C., 2015. RSC Adv. 5(95), 77755-77759.

Yang, M.Z., Zhang, W., Yang, J.C., Hu, B.F., Cao, F.J., Zheng, W.S., Chen, Y.P., Jiang, X.Y., 2017. Sci. Adv. 3(12), 6.

Yih, L.H., Lee, T.C., 2000. Cancer Res. 60(22), 6346-6352.

Zhan, S., Yu, M., Lv, J., Wang, L., Zhou, Pei., 2014. Aust. J. Chem. 67(5), 813-818.

Yuan, M., Zhang, Q., Song, Z., Ye, T., Yu, J., Cao, H., Xu, F., 2019. Microchim. Acta 186(5), 268.

Zeng, L., Zhou, D., Gong, J., Liu, C., Chen, J., 2019. Anal. Chem. 91(3), 1724-1727.

Zhan, S., Wu, Y., Wang, L., Zhan, X., Zhou, P., 2016. Biosens. Bioelectron. 86, 353368.

Zhang, H., Ma, X., Liu, Y., Duan, N., Wu, S., Wang, Z., Xu, B., 2015. Biosens. Bioelectron. 74, 872-877.

Zhang, L., Cheng, X.Z., Kuang, L., Xu, A.Z., Liang, R.-P., Qiu, J.D., 2017a. Biosens. Bioelectron. 94, 701-706.

Zhang, S., Geryak, R., Geldmeier, J., Kim, S., Tsukruk, V.V., 2017b. Chem. Rev. 117(20), 12942-13038.

Zhang, Z., Shikha, S., Liu, J., Zhang, J., Mei, Q., Zhang, Y., 2019. Anal. Chem. 91(1), 548-568.

Zhao, Y., Chen, F., Li, Q., Wang, L., Fan, C., 2015. Chem. Rev. 115(22), 12491-12545. Zhou, Q., Tang, D., 2018. J. Mater. Chem. B 6(41), 6585-6591.

Zuo, X., Song, S., Zhang, J., Pan, D., Wang, L., Fan, C., 2007. J. Am. Chem. Soc. 129(5), 1042-1043. 